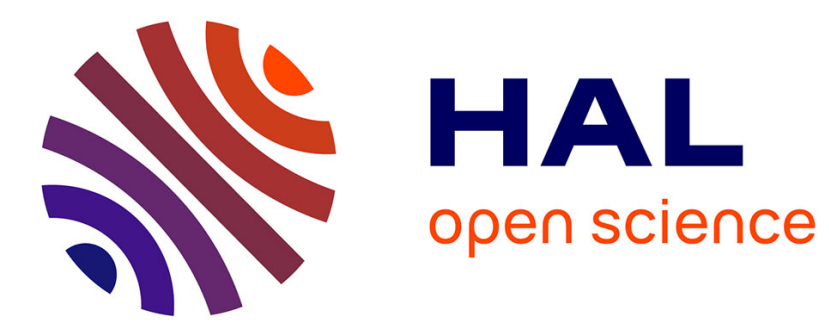

\title{
Measuring anisotropy of elastic wave velocity with ultrasound imaging and an autofocus method: application to cortical bone
}

Guillaume Renaud, Pierre Clouzet, Didier Cassereau, Maryline Talmant

\section{To cite this version:}

Guillaume Renaud, Pierre Clouzet, Didier Cassereau, Maryline Talmant. Measuring anisotropy of elastic wave velocity with ultrasound imaging and an autofocus method: application to cortical bone. Physics in Medicine and Biology, 2020, 10.1088/1361-6560/abb92c . hal-02989381

\section{HAL Id: hal-02989381 \\ https://hal.science/hal-02989381}

Submitted on 5 Nov 2020

HAL is a multi-disciplinary open access archive for the deposit and dissemination of scientific research documents, whether they are published or not. The documents may come from teaching and research institutions in France or abroad, or from public or private research centers.
L'archive ouverte pluridisciplinaire HAL, est destinée au dépôt et à la diffusion de documents scientifiques de niveau recherche, publiés ou non, émanant des établissements d'enseignement et de recherche français ou étrangers, des laboratoires publics ou privés. 


\title{
Measuring anisotropy of elastic wave velocity with ultrasound imaging and an autofocus
}

\section{method - Application to cortical bone}

\author{
Guillaume Renaud*, Pierre Clouzet*, Didier Cassereau*, and Maryline Talmant* \\ *Sorbonne Université, CNRS UMR 7371, INSERM UMR S 1146, Laboratoire d'Imagerie Biomédicale, \\ Paris, France
}

\begin{abstract}
This work investigates the feasibility of estimating the parameters of an exact transverse isotropy model in cortical bone. The model describes the anisotropy of the velocity of compressional and shear bulk elastic waves. We propose to achieve this with ultrasound imaging relying on the transmission of unfocused beams and with an autofocus method. The latter is based on the principle that the reconstructed ultrasound image shows optimal quality if the velocity model is correct. The autofocus approach is applied to a composite image of the interface between cortical bone and marrow. It is obtained by incoherent summation of 4 types of images exploiting 4 different ray paths in the cortical bone layer, 3 of them involving mode-converted shear waves. If the parameters of the model are correct, spatial co-localization of the interface appears in the four images. As a result, intensity and sharpness in the composite image are maximal.

The five parameters of the model of transverse isotropy are successfully estimated in a tube made of a bone-mimicking material. The estimates are in good agreement with resonant ultrasound spectroscopy measurements. The tube thickness is recovered with an error smaller than $0.3 \%$. In vivo results at the forearm of a volunteer are promising, four parameters could be estimated and are in good agreement with ex vivo resonant ultrasound spectroscopy measurements. Moreover X-ray peripheral computed tomography corroborates the thickness of the cortical bone layer in the ultrasound image. Weak-anisotropy and exact transverse isotropy models provide very close measurements of the thickness of the tube and the radius bone. Thus, we recommend using the model of weak transverse isotropy for real-time anatomical imaging because more computationally efficient. For material characterization however, the model of exact transverse isotropy is preferred because the elastic anisotropy of cortical bone is moderate, rather than weak.
\end{abstract}

\section{Index Terms}

Ultrasound imaging, cortical bone, refraction, ray tracing, elastic anisotropy, autofocus.

Corresponding Author: Guillaume Renaud, Laboratoire d'Imagerie Biomédicale, 15 rue de l'école de médecine, 75006 Paris, France; Email, guillaume.renaud@upmc.fr; Phone, +331442792 24. 


\section{INTRODUCTION}

Achieving absolute quantitative measurement of the compressional wave-speed in biological tissues in vivo has been a topic of interest since the 1970's, in particular with the early developments of ultrasound tomography [1], [2]. Ultrasound tomography is now a mature technology [3], [4], however it requires dedicated equipment. On the other hand, single-sided hand held ultrasound imaging is spread worldwide and associated analyses of the echo signals were shown to be capable of measuring the compressional wave-speed in a homogeneous region of tissue with isotropic acoustic properties [5]-[9]. Ongoing research is aiming at producing a parametric image of the compressional wave-speed [10]-[13], just like ultrasound tomography, but with hand held ultrasound imaging. The shear wave-speed can also be measured in soft tissues with transient ultrasound elastography [14]. The absolute measurement of the speed of sound (shear and compressional waves) has proven to be successful for non-invasive detection and staging of diseases, like fibrosis, steatosis or cancer [3], [7], [15].

In the 1970's, the ultrasonic wave-speed was proposed as a biomarker of the quality of cortical bone tissue [16], [17]. The measurement of the elastic anisotropy of cortical bone in vivo with ultrasound has been only recently addressed [18]-[20]. Assessing the anisotropy of elastic wave velocities in cortical bone is of interest, because it provides information on the nano- and micro-structure of cortical bone tissue. Indeed the elastic anisotropy of cortical bone sensed by ultrasound in the 1-5 MHz range (wavelength in the 0.5-4 mm range) is determined at two scales by two features, namely the orientation of mineralized collagen fibers constituting the solid phase of bone and the Haversian canals (created by bone remodeling) hosting blood vessels and nerves [21]. In the cortex of the diaphysis of a long bone, the Haversian canals (approximately cylindrical pores, $\sim 50 \mu m$ in diameter) are nearly aligned with the axis of the long bone, which causes elastic anisotropy at the scale of few millimeters [22]. In addition, the complicated organization of mineralized collagen fibers results in elastic anisotropy at the scale of few hundreds of micrometers [23], [24], which also contributes to elastic anisotropy at the scale of few millimeters. In a recent publication [20], we showed that the cortex of a long bone can be imaged in vivo with single-sided

hand held ultrasound imaging if elastic anisotropy is considered. The region of interest was regarded as a layered medium, and refraction occurring at the interface between layers was accounted for during image reconstruction. In [20], we demonstrated that a model of weak transverse isotropy proposed by seismologists [25] was suitable and the thickness of the bone cortex in the ultrasound image was in agreement with x-ray computed tomography. The compressional wave-speed and its anisotropy were estimated in the cortical bone layer with an "autofocus approach" [26].

Like in [20], this work capitalizes on the autofocus method [26]. Its rationale is simple: the reconstructed ultrasound image shows optimal quality (intensity and sharpness) if the values taken by the parameters of the wave-speed model are correct. This concept was first introduced in medical ultrasound for aberration correction in soft tissues [6], [27]-[29] and later in medical photoacoustic imaging [26]. In this early work, the goal was to 
improve image quality and the targets in the medium were small compared to the acoustic wavelength (diffuse scattering). Here, we apply the autofocus approach to specular reflections at the interfaces between a cortical bone layer and soft tissues and our primary goal is the estimation of the anisotropy of compressional and shear wavespeeds in cortical bone. Compared to our previous publication [20], we introduce here two new features: we will use a model of exact transverse isotropy (while a model of weak transverse isotropy was used in [20]) and we will introduce the concept of composite image constructed as an incoherent summation of 4 types of images exploiting 4 different ray paths, 3 of them involving mode-converted shear waves (while images in [20] were only based on compressional waves). The feasibility of estimating experimentally the parameters of the model of exact transverse isotropy with a composite ultrasound image is explored. The approach is tested first on a tube made of a bonemimicking fiber-reinforced composite material, then in vivo at the forearm of a healthy volunteer. The added value of using an exact model of transverse isotropy for image reconstruction and thickness measurement, compared to the approximated model of weak transverse isotropy used in [20], is also evaluated.

\section{WAVE-SPEED ANISOTROPY IN HUMAN CORTICAL BONE}

The elasticity of human cortical bone at the middle tubular part of a long bone (diaphysis) is well described with a model of transverse isotropy (or hexagonal symmetry) [30]-[32]. This means that human cortical bone possesses a plane of isotropy in which the velocity of a compressional or shear wave does not depend on the propagation direction. This plane of isotropy is normal to the axis of osteons (structures created by bone remodeling, each osteon contains a quasi-cylindrical pore called haversian canal). At the diaphysis of a long bone, the axis of osteons is nearly aligned with the axis the long bone. Three types of bulk elastic waves are encountered in such a material for a propagation direction in a plane containing the axis of the long bone: a quasi-compressional wave with quasilongitudinal polarization (or quasi P-wave), a quasi-shear wave with quasi-vertical polarization (or quasi SV-wave), and a pure shear wave with horizontal polarization (or SH-wave).

The link between phase velocities of bulk elastic waves and the class of symmetry of the material is well known. Conventional formulation of the phase velocities involves elastic stiffnesses $C_{i j}$ (5 elastic constants for transverse isotropy) and mass density $\rho$ [33]. There exist other formulations of the phase velocities of bulk elastic waves. In particular, Thomsen [25] proposed to recast the expressions of phase velocities of bulk elastic waves using wave-speeds in specific orientations. In this work, we adopt Thomsen's formulation.

\section{A. Thomsen's formulation for exact transverse isotropy}

In Thomsen's expressions of the compressional phase velocity $v_{P}$ and the shear phase velocity with vertical polarization $v_{S V}$, two parameters of anisotropy called $\epsilon$ and $\delta^{*}$ appear in addition to the phase velocity of a compressional wave in the direction of the pores (i.e. along the axis of a long bone) termed $\alpha_{0}$ and the phase velocity of a shear wave with vertical polarization in the direction of the bone axis (or normal to this direction) 
termed $\beta_{0}$. The shear phase velocity with horizontal polarization $v_{S H}$ is determined by the two velocities $\beta_{0}$ and $V_{S H}^{\text {radial }}$. Thomen's expressions [25] are recalled below:

$$
\begin{aligned}
& v_{P}(\theta)=\alpha_{0}\left[1+\epsilon \cos ^{2}(\theta)+D^{*}\left(\theta, \alpha_{0}, \beta_{0}, \epsilon, \delta^{*}\right)\right]^{1 / 2} \\
& v_{S V}(\theta)=\beta_{0}\left[1+\left(\frac{\alpha_{0}^{2}}{\beta_{0}^{2}}\right)\left(\epsilon \cos ^{2}(\theta)-D^{*}\left(\theta, \alpha_{0}, \beta_{0}, \epsilon, \delta^{*}\right)\right)\right]^{1 / 2} \\
& v_{S H}(\theta)=\beta_{0}\left[1+\frac{V_{S H}^{\text {radial } 2}-\beta_{0}^{2}}{\beta_{0}^{2}} \cos ^{2}(\theta)\right]^{1 / 2} \\
& D^{*}=\frac{1}{2}\left(1-\frac{\beta_{0}^{2}}{\alpha_{0}^{2}}\right)\left[\left(1+\frac{4 \delta^{*}}{\left(1-\beta_{0}^{2} / \alpha_{0}^{2}\right)^{2}} \cos ^{2}(\theta) \sin ^{2}(\theta)+\frac{4\left(1-\beta_{0}^{2} / \alpha_{0}^{2}+\epsilon\right) \epsilon}{\left(1-\beta_{0}^{2} / \alpha_{0}^{2}\right)^{2}} \cos ^{4}(\theta)\right)^{1 / 2}-1\right] \\
& \alpha_{0}=\sqrt{\frac{C_{33}}{\rho}} \\
& \beta_{0}=\sqrt{\frac{C_{44}}{\rho}} \\
& \epsilon=\frac{C_{11}-C_{33}}{2 C_{33}} \\
& \delta^{*}=\frac{1}{2 C_{33}^{2}}\left(2\left(C_{13}+C_{44}\right)^{2}-\left(C_{33}-C_{44}\right)\left(C_{11}+C_{33}-2 C_{44}\right)\right) \\
& V_{S H}^{\text {radial }}=\sqrt{\frac{C_{66}}{\rho}}
\end{aligned}
$$

$\theta$ is the phase angle between the wave vector and the normal to the axis of the long bone. We recall that, with transverse isotropy, $C_{11}=C_{22}, C_{44}=C_{55}, C_{23}=C_{13}$ and $C_{12}=C_{11}-2 C_{66}$. For ultrasound image reconstruction, one needs the group velocity $V$ (ultrasound ray velocity) and group angle $\phi$ (ultrasound ray angle), they can be calculated as follows [25]:

$$
\begin{aligned}
\tan (\phi(\theta)) & =\left(\tan \theta+\frac{1}{v} \frac{\partial v}{\partial \theta}\right) /\left(1-\frac{\tan \theta}{v} \frac{\partial v}{\partial \theta}\right) \\
V^{2}(\phi(\theta)) & =v^{2}(\theta)+\left(\frac{\partial v}{\partial \theta}\right)^{2}
\end{aligned}
$$

\section{B. Weak elastic anisotropy (transverse isotropy)}

Thomsen's formulation is particularly popular when simplified in the case of weak anisotropy [25]. This weakanisotropy model assumes $\left|\delta^{*}\right| \ll 1$ and $|\epsilon| \ll 1$, it is obtained after full linearization of equations 1 to 4 . We used this model in our previous publication [20], for describing the group velocity of a compressional wave in cortical bone. The group velocity of a compressional wave $V_{P}$, a vertically-polarized shear wave $V_{S V}$ and a shear wave with horizontal polarization $V_{S H}$ can be written as: 


$$
\begin{aligned}
V_{P}(\phi) & =V_{P}^{\text {axial }}-\left(V_{P}^{\text {axial }}-V_{P}^{\text {radial }}\right) \times\left[\xi \sin ^{2}(\phi) \cos ^{2}(\phi)+\cos ^{4}(\phi)\right] \\
V_{S V}(\phi) & =\beta_{0}\left[1+\sigma \sin ^{2}(\phi) \cos ^{2}(\phi)\right] \\
V_{S H}(\phi) & =\beta_{0}\left[1+\frac{V_{S H}^{\text {radial } 2}-\beta_{0}^{2}}{2 \beta_{0}^{2}} \cos ^{2}(\phi)\right]
\end{aligned}
$$

$V_{P}^{\text {radial }}=\sqrt{C_{11} / \rho}$ is the compressional wave-speed in the plane of isotropy. $V_{P}^{\text {axial }}=\alpha_{0}=\sqrt{C_{33} / \rho}$ is the compressional wave-speed in the direction of the axis of the long bone. $\xi$ is an anisotropy form parameter introduced in [20] (note that $\xi$ was called $\beta$ in [20], this change is to avoid confusion with Thomsen's parameter $\beta_{0}$ ).

\section{Values of Thomsen's parameters for human cortical bone}

Let us now investigate what values take Thomsen's parameters for human cortical bone. To do this, we used $e x$ vivo resonant ultrasound spectroscopy measurements reported in the literature [32], [34], [35] to calculate $\alpha_{0}, \beta_{0}$, $\epsilon$ and $\delta^{*}$. From all the samples we selected those with a high mass density, namely between 1900 and $2000 \mathrm{~kg} / \mathrm{m}^{3}$ (corresponding to a porosity of approximately 5\%), because the in vivo feasibility in this work is conducted on a healthy volunteer. The selected 87 samples were taken from the tibia, the femur or the radius of donors (50-95 years old). Figure 1 shows the values of Thomsen's parameters for these 87 samples of human cortical bone.

The median values of $\alpha_{0}, \beta_{0}$ and $V_{S H}^{\text {radial }}$ for human cortical bone with high mass density are $3.9 \mathrm{~mm} / \mu \mathrm{s}$, $1.8 \mathrm{~mm} / \mu \mathrm{s}$ and $1.5 \mathrm{~mm} / \mu \mathrm{s}$, respectively. As a result of the oriented porosity in haversian human cortical bone (cylindrical pores nearly aligned with the axis of a long bone) [31], $\epsilon<0$ since the compressional phase velocity for $\theta=0^{\circ}$ (i.e. $V_{P}^{\text {radial }}$ ) is smaller than that at $\theta=90^{\circ}$ (i.e. $V_{P}^{\text {axial }}$ or $\alpha_{0}$ ). The median value of $\epsilon$ and $\delta^{*}$ is -0.17 and -0.15 , respectively. Therefore the elastic anisotropy of high-density human cortical bone is moderate but not exactly small. For this reason, even if equation 12 can efficiently fit the exact relation between the compressional group velocity and the group angle [36], the relation between the anisotropy form parameter $\xi$ and the elastic constants is lost. However, because the weak anisotropy model for a compressional wave reduces to a simple formula with only 3 parameters, equation 12 is very useful for reducing computational time and therefore enabling real-time ultrasound imaging of bone with several images per second. Moreover we showed in [20] that the model of weak anisotropy for a compressional wave produced an image of the bone cortex in agreement with high-resolution x-ray computed tomography.

Thus our motivation in this work is to investigate the model of exact transverse isotropy for accurate characterization of the elasticity of cortical bone, which cannot be precisely performed with a model of weak elastic anisotropy.

\section{Expected variations of the anisotropy of $P, S V$ and $S H$ group velocities for high-density human cortical bone}

It is seen from equations 1 and 4 that the magnitude of anisotropy on the phase velocity (and group velocity) for a compressional wave is determined by $\epsilon$. Indeed, for $\theta=0^{\circ}$ (direction of propagation normal to axis of the long bone), $v_{P}\left(\theta=0^{\circ}\right)=\alpha_{0} \sqrt{1+2 \epsilon}=\sqrt{C_{11} / \rho}$. Figure 2 shows numerical calculations of $\mathrm{P}, \mathrm{SV}$ and SH 

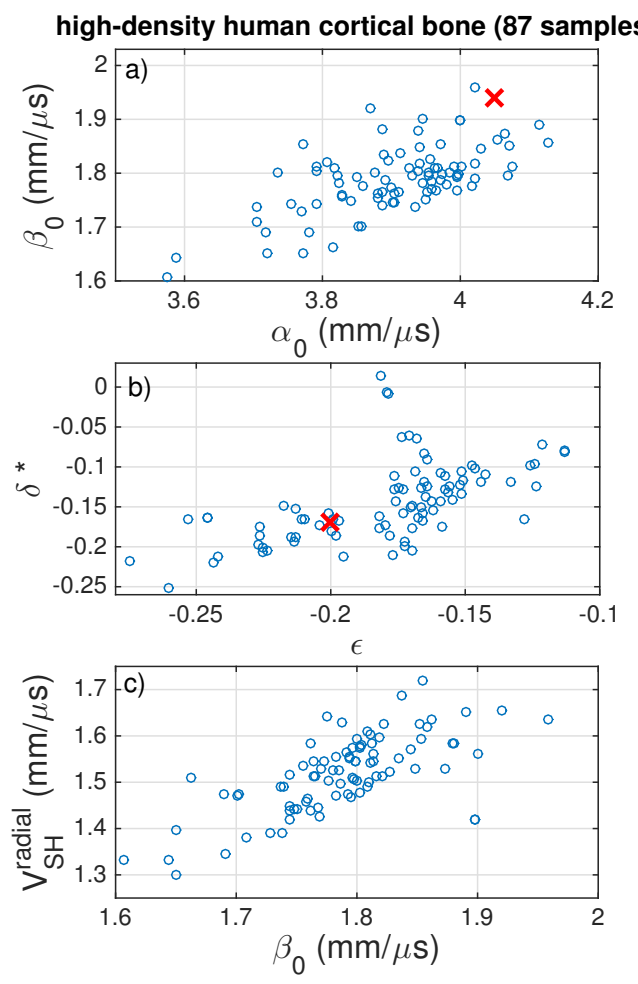

Fig. 1. Distribution of values of Thomsen's parameters calculated from ex vivo resonant ultrasound spectroscopy measurements [32], [34], [35]. A selection of 87 samples with a high mass density between 1900 and $2000 \mathrm{~kg} / \mathrm{m}^{3}$ was used, because the in vivo feasibility in this work is conducted on a healthy volunteer. The estimates obtained in vivo in this work (see section VI) are shown with a red cross.

group velocities using equations 1 to 11 . It is seen that $\delta^{*}$ and $\beta_{0}$ only influence the form of the anisotropy for a compressional wave. Moreover it is clear that the influence of $\delta^{*}$ is much larger than that of $\beta_{0}$ (within the expected range of values for high-density human cortical bone).

For a vertically-polarized shear wave, it is seen from equations 2 and 4 that $v_{S V}\left(\theta=0^{\circ}\right)=v_{S V}\left(\theta=90^{\circ}\right)=\beta_{0}$. The magnitude and form of the anisotropy for a vertically-polarized shear wave is determined by $\delta^{*}, \epsilon$ and $\alpha_{0}$ (Figure 2). However the influence of $\alpha_{0}$ is weak compared to that of the two other parameters. Depending on the values of $\delta^{*}$ and $\epsilon$, the velocity of a vertically-polarized shear wave for an angle between $0^{\circ}$ and $90^{\circ}$ can be larger or smaller than $\beta_{0}$, and the maximum change of wave-speed is observed for an angle close to $40^{\circ}$.

The group velocity of a horizontally-polarized shear wave is minimum for $\theta=0^{\circ}$ and equals $V_{S H}^{\text {radial }}=\sqrt{C_{66} / \rho}$ (Figure 2). It is maximum for $\theta=90^{\circ}$ and equals $\beta_{0}$. 


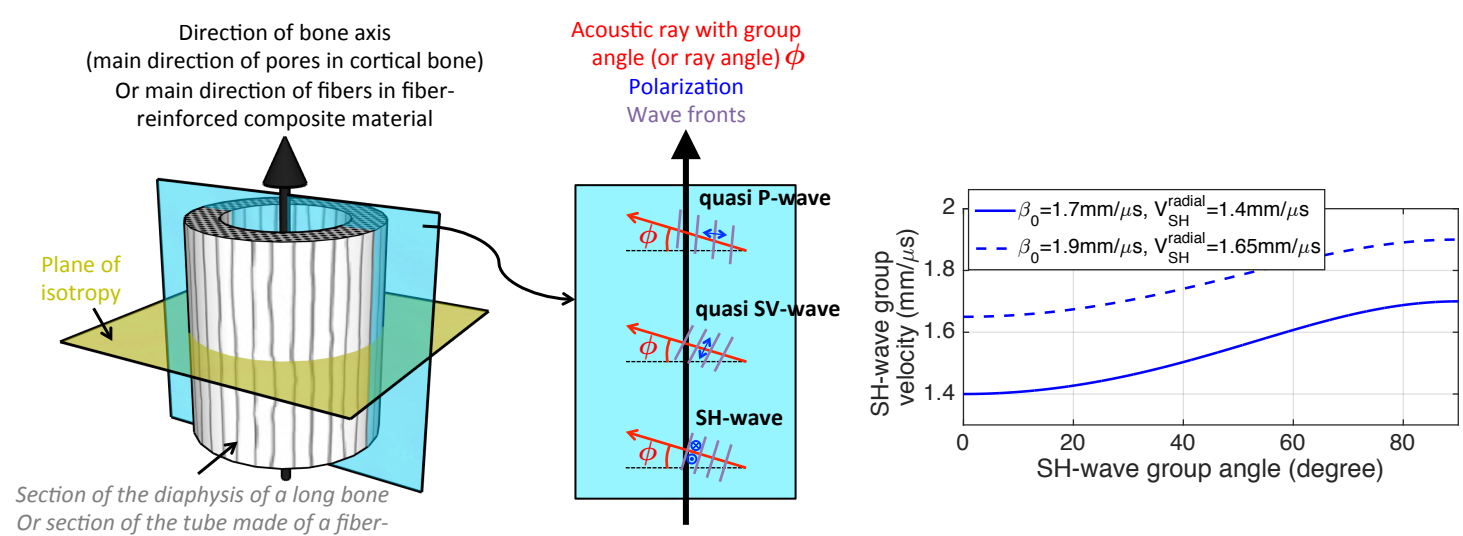

reinforced composite material


Fig. 2. Numerical calculations of $\mathrm{P}, \mathrm{SV}$ and $\mathrm{SH}$ group velocities using equations 1 to 11 . Variation of the group velocity of a compressional wave (P-wave, left column), a vertically-polarized shear wave (SV-wave, right column) and a horizontally-polarized shear wave (SH-wave, top right panel), for expected values of $\alpha_{0}, \beta_{0}, \delta^{*}, \epsilon$ and $V_{S H}^{\text {radial }}$ in high-density human cortical bone (see Figure 1). When a parameter is kept constant, the median value calculated from resonant ultrasound spectroscopy measurement for high-density human cortical bone is used.

\section{JOINT PARAMETER ESTIMATION WITH ULTRASOUND IMAGING AND THE AUTOFOCUS APPROACH}

We consider here the recording the ultrasound echo signals using a conventional clinical ultrasound probe. Such a probe is most often made of a linear array of independent piezoelectric elements, able to generate an outgoing ultrasound wave and record backscattered ultrasound waves. The ultrasound probe is placed close to the elastic layer we aim to study (distance close to $5 \mathrm{~mm}$ ). Figure 3 illustrates the four types of ray paths considered in this 
work, when the ultrasound transducer is positioned for a longitudinal 2D image or a transverse 2D image:

- the "PP" path: reflection of the compressional wave broadcasted by the emitting element at the outer surface of the elastic layer

- the "PPPP" path: reflection of the compressional wave broadcasted by the emitting element at the inner surface of the elastic layer, without mode conversion

- the "PSSP" path: reflection of the compressional wave broadcasted by the emitting element at the inner surface of the elastic layer, without mode conversion during reflection but with mode conversion (compressional wave to shear wave and then shear wave to compressional wave) during transmission/refraction at the outer surface of the elastic layer

- the "PSPP/PPSP" path: reflection of the compressional wave broadcasted by the emitting element at the inner surface of the elastic layer, with mode conversion during reflection and during transmission/refraction at the outer surface of the elastic layer (compressional wave to shear wave or shear wave to compressional wave)

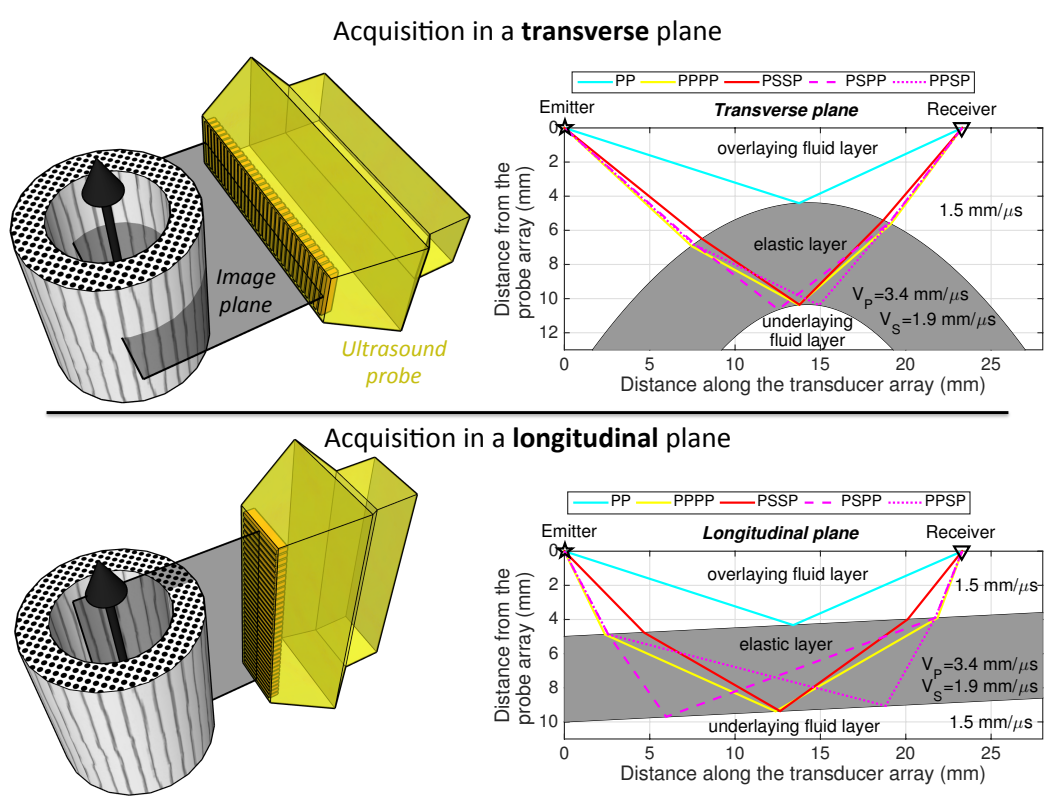

Fig. 3. Illustration of the two acquisition configurations and the ray paths considered in this work; in a transverse plane (top) and in a longitudinal plane (bottom) of a tubular object. The situation depicted in the figure corresponds to a transmission with an element of the probe array and reception with a different element. The four paths are labeled with $P$ and $S$ referring to a compressional wave and a shear wave, respectively. In this illustration, the elastic layer has isotropic elasticity with a compressional wave-speed of $3.4 \mathrm{~mm} / \mu \mathrm{s}$ and a shear wave-speed of $1.9 \mathrm{~mm} / \mu \mathrm{s}$. The speed of sound in the overlaying and underlaying fluid layers is $1.5 \mathrm{~mm} / \mu \mathrm{s}$. 
It is important to note that the mode-converted shear wave is expected to have a vertical polarization with an acquisition in a longitudinal plane. With an acquisition in a transverse plane, the mode-converted shear wave is expected to have a horizontal polarization.

In this work, instead of considering the PPPP path only, like in [20], we wish to exploit all the information carried by the four types of path to estimate the parameters of the model of exact transverse isotropy. In fact, an ultrasound image can be reconstructed for each of the four types of ray path. The information contained in the four types of images is further processed after summation of the four normalized envelope images (incoherent compounding), which results in a single composite image:

$$
\begin{aligned}
\text { Composite Image }= & \text { normalized PPPP image }+ \text { normalized PSSP image }+ \\
& \text { (normalized PSPP image }+ \text { normalized PPSP image }) / 2
\end{aligned}
$$

In the configurations studied in this work, the imaged object has locally a tubular shape and the thickness of the elastic layer is nearly constant in the region of interest. Moreover the elastic anisotropy is moderate. As a result, the travel times of PSPP and PPSP paths are nearly equal, and hence PSPP and PPSP images are nearly identical. Thus the PSPP and PPSP images are weighted by a factor 0.5 when constructing the composite image (Equation 15). It is important to note that the purpose of constructing such a composite image is two-fold:

- searching for maximum quality (intensity and sharpness) in the four image types simultaneously by working with a single composite image containing information from the four wave paths PPPP, PSPP, PPSP and PSSP

- searching for spatial co-localization of the interface between the solid elastic layer and the underlaying fluid layer in the four types of image, because PPPP, PSPP, PPSP and PSSP paths all include a reflection at the same interface

Perhaps surprisingly, it will be seen that the composite image does not improve image quality, compared to a PPPP image (Figures 6, 7 and 8). A PPPP image exhibits better contrast for seeing the interface between the solid elastic layer and the underlaying fluid layer, while the composite image appears blurry. That is why the thickness of the elastic layer will be measured in PPPP images. The purpose of the composite image is not to improve image quality, we propose to use it for estimating the parameters of the model of exact transverse isotropy.

\section{A. Strategy for estimating the thickness of an elastic layer and the anisotropy of wave-speeds}

We propose the following 7-step strategy to evaluate the five parameters of the model of exact transverse isotropy $\left(\alpha_{0}, \epsilon, \delta^{*}, \beta_{0}\right.$ and $\left.V_{S H}^{\text {radial }}\right)$ and estimate the thickness of the solid elastic layer:

1) Estimation of the speed of sound in the first fluid layer (between the ultrasound probe and the solid elastic layer) with the autofocus method. The region selected to calculate the metrics of image quality encompasses the outer surface of the solid elastic layer

2) Reconstruction of the image from the ultrasound probe to the outer surface of the solid elastic layer with the optimal speed of sound in the overlaying fluid layer found with step 1 
3) Segmentation of the first interface between the overlaying fluid layer and the solid elastic layer using a technique based on Dijkstra's algorithm, the result of the segmentation is approximated by a parabola

4) Estimation of velocity model in the elastic layer:

a) With an acquisition in a plane of isotropy (transverse plane, i.e. normal to bone axis or normal to fiber direction in bone-mimicking material), reconstruction of composite images with compressional and horizontally-polarized shear wave velocity models (PPPP, PSPP, PPSP, PSSP paths), estimation of $V_{P}^{\text {radial }}$ and $V_{S H}^{\text {radial }}$ with the autofocus method on the composite images. The composite image being constructed as the sum of the normalized envelopes of the four types of images (incoherent compounding). The region selected to calculate the metrics of image quality encompasses the interface between the solid elastic layer and the underlaying fluid layer. The optimal values of $V_{P}^{\text {radial }}$ and $V_{S H}^{\text {radial }}$ are obtained with a two-dimensional grid search

b) With an acquisition in a longitudinal plane (parallel to bone axis or parallel to fiber direction in bonemimicking material), estimation of $V_{P}^{a x i a l}\left(\right.$ i.e. $\left.\alpha_{0}\right)$ with the head-wave velocity

c) From steps $4 \mathrm{a}$ and $4 \mathrm{~b}, \epsilon$ can be calculated since $\epsilon=\left(V_{P}^{\text {radial } 2}-\alpha_{0}^{2}\right) /\left(2 \alpha_{0}^{2}\right)$

d) With an acquisition in a longitudinal plane (parallel to bone axis or parallel to fiber direction in bonemimicking material), reconstruction of composite images with compressional and vertically-polarized shear wave velocity models (PPPP, PSPP, PPSP, PSSP paths), estimation of $\delta^{*}$ and $\beta_{0}$ with the autofocus method on the composite images. The composite image being constructed as the sum of the normalized envelopes of the four types of images (incoherent compounding). The region selected to calculate the metrics of image quality encompasses the interface between the solid elastic layer and the underlaying fluid layer. The optimal values of $\delta^{*}$ and $\beta_{0}$ are obtained with a two-dimensional grid search

\section{5) Measurement of thickness of the elastic layer with the PPPP image:}

a) Reconstruction of the image using the PPPP path from the beginning to the end of the solid elastic layer with the optimal four parameter values of the model for exact transverse isotropy found in steps $4 \mathrm{a}$ to $4 \mathrm{~d}$

b) Segmentation of the second interface between the solid elastic layer and the underlaying fluid layer using a technique based on Dijkstra's algorithm, the result of the segmentation is approximated by a parabola

c) The thickness is calculated as the mean distance between the two segmented interfaces (steps 3 and 5b), details are provided in Appendix C

It is worth noticing that steps 1 to 3 , step $4 \mathrm{~b}$ and steps $5 \mathrm{a}$ to $5 \mathrm{c}$ were introduced in our previous publication [20]. The novelty here lies in steps $4 \mathrm{a}$ and $4 \mathrm{~d}$, i.e. the construction and usage of a composite image. Step 1 will not be addressed in this manuscript, since the procedure was described in [20].

The details of step 4 (estimation of the parameters of the velocity model in the solid layer with anisotropic elasticity) 
are graphically illustrated in Figure 4.

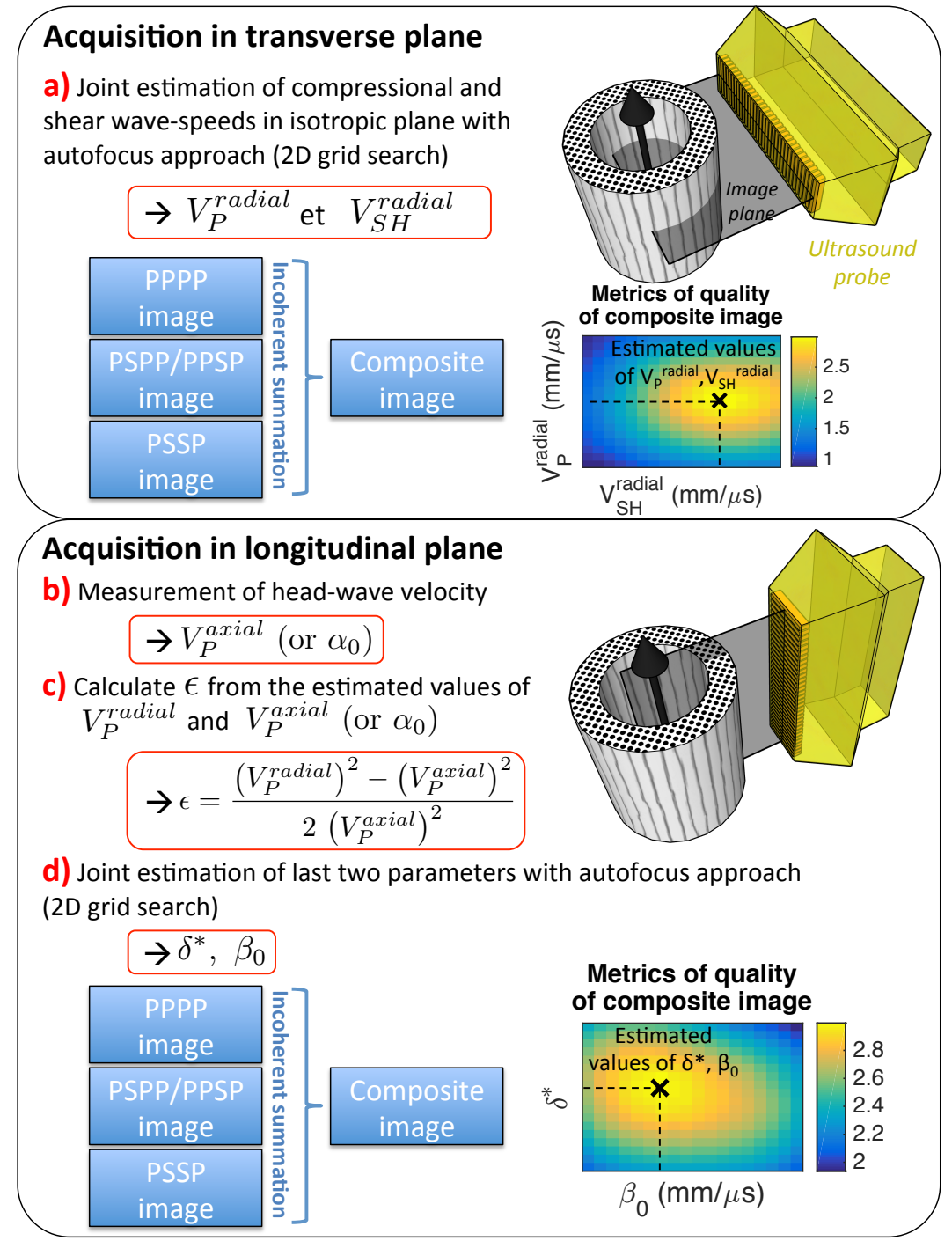

Fig. 4. Illustration of the strategy to estimate the five parameters of the model of exact transverse isotropy.

In order to evaluate the added value of the model of exact transverse isotropy versus a model of weak anisotropy, we also apply in this work the procedure proposed in [20] for PPPP images. The velocity model of a compressional wave for weak anisotropy (equation 12) has three parameters: $V_{P}^{a x i a l}\left(\right.$ or $\left.\alpha_{0}\right), V_{P}^{\text {radial }}$ and $\xi$. The corresponding procedure is close to the strategy proposed above for exact transverse isotropy. However step 4a estimates $V_{P}^{\text {radial }}$ only (one-dimensional grid search), and steps $4 \mathrm{c}$ and $4 \mathrm{~d}$ are replaced with a single step that estimates $\xi$ (onedimensional grid search). 


\section{B. Ultrasound acquisitions and image reconstruction}

Like in [20] ultrasound data is acquired with an ultrasound transducer array positioned in two configurations (Figure 3). For the ultrasound data acquisition we used a fully programmable ultrasound system (Vantage, Verasonics Inc., Redmond, WA, USA) equipped with a $2.5 \mathrm{MHz}$ linear phased-array transducer (P4-1 ATL/Philips, Bothell, WA, USA; pitch $0.295 \mathrm{~mm}, 96$ elements). A synthetic aperture transmit scheme [37] was employed, in which every single element in the array subsequently transmits a short ultrasound pulse, followed by a full array recording of the backscattered echo signals. Because the element width is approximately half the wavelength, a single element broadcasts a quasi-spherical diverging wavefront. Synthetic aperture ultrasound imaging consists in reconstructing 96 low-resolution images (because 96 shots with 96 individual elements), then the low-resolution images are summed (coherent compounding) to form a high-resolution image [38]. Each low-resolution image is reconstructed with a delay-and-sum algorithm. Finally the envelope of the high-resolution image is computed. Details on the image reconstruction algorithm are provided in Appendix A.

\section{The autofocus method: Estimation of wave-speed by searching for maximum image intensity and sharpness}

The estimation of all wave-speed parameters except $\alpha_{0}$ (estimated with the velocity of the head-wave, see Appendix B) is based on the fact that the reconstructed image shows optimal quality if the values taken by the parameters of the velocity model are correct. Optimal image quality can be obtained by searching for maximum intensity and maximum sharpness of the image [20], [26]. Two metrics of image sharpness are investigated in this work: the normalized variance and the Brenner sharpness [26]. Maximum image quality is searched with a grid-search approach (of course an optimization method like the downhill simplex method [39] can be implemented to reduce processing time). For a given parameter, an interval of search is determined based on prior knowledge. Typically 20 values in the interval are tested, for each value an image is reconstructed and metrics of image quality are calculated. The three metrics obtained for the 20 tested values are then normalized by their maximum values and summed. Finally the estimate of a given parameter is the value for which the sum of the three normalized metrics is maximal.

Figure 5 illustrates the rationale of the method with synthetic signals in the case of the reflection by a curved interface. Two sets of echo signals were generated, corresponding to two separate transmissions by two single elements of a transducer array. Note that the work reported here uses 96 transmissions, here we illustrate the autofocus method with only two transmission for clarity. Two low-resolution images are reconstructed and then added to create a high-resolution image. It is seen that the two transmissions capture different parts of the interface, however the two parts overlap. As a result, when the two low-resolution images are summed (coherent compounding), constructive or destructive interferences can happen. Optimal constructive interferences between the two lowresolution images occur only if the wave-speed is correct, and maximum intensity and sharpness is observed in the envelope of the high-resolution image. Moreover, the correct spatial position of the interface is only recovered if the wave-speed is correct.

In fact, the autofocus approach is similar to Migration Velocity Analysis (MVA) in seismology (see for instance 
[40], note that migration is a synonym for image reconstruction). The objective of MVA is the estimation of a velocity model in the image domain, it is based on the principle that images (for example a region encompassing an interface) reconstructed with different sources and/or receivers should be consistent if the velocity model is correct.

\section{Wave-speed too low}

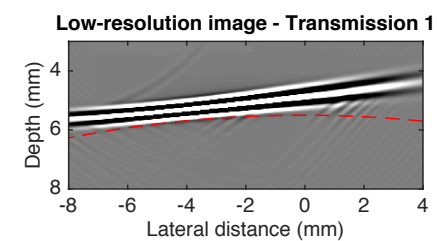

Low-resolution image - Transmission 2

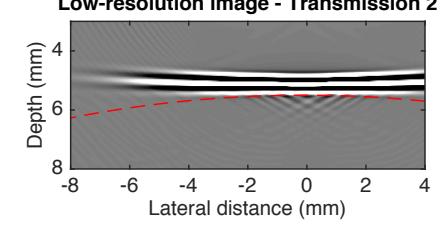

High-resolution image (Coherent compounding
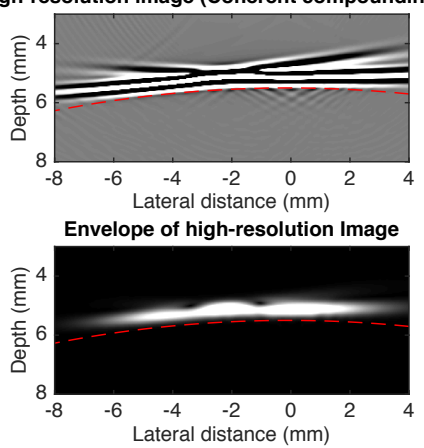

Depth underestimated Suboptimal intensity Suboptimal sharpness

\section{Correct wave-speed}

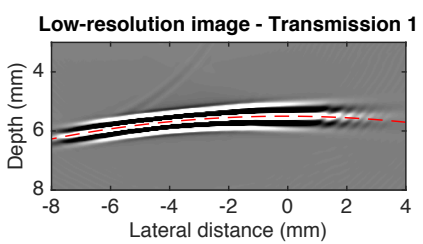

Low-resolution image - Transmission 2
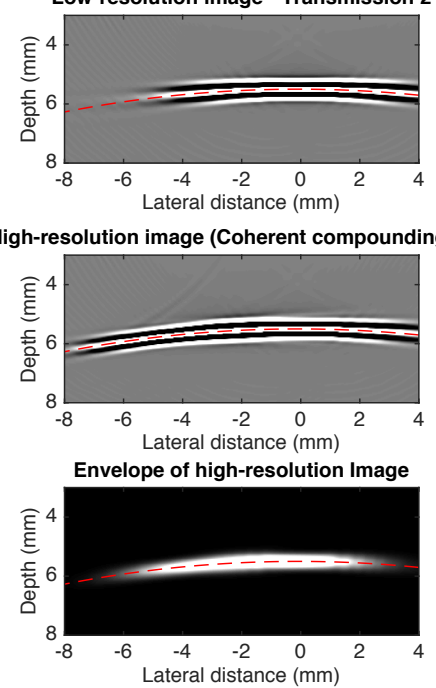

Correct position

Maximum intensity

Maximum sharpness

\section{Wave-speed too high}
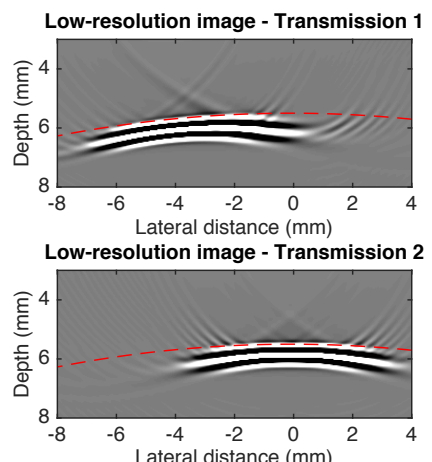

igh-resolution image (Coherent compounding 1+2)

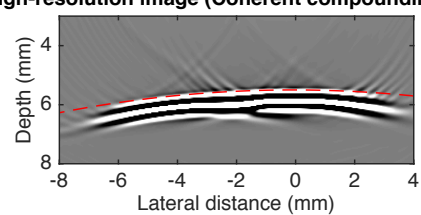

Envelope of high-resolution Image

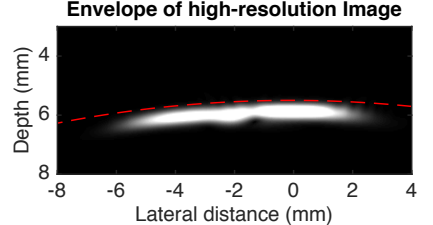

Depth overestimated

Suboptimal intensity

Suboptimal sharpness

Fig. 5. Illustration of the autofocus method with only two transmissions by two separate single-elements of the transducer array. Synthetic echo signals were generated in the case of the reflection by a curved interface. The true position of the interface is depicted in red. When the two low-resolution images are added (coherent compounding), construction or destructive interferences can happen. Optimal constructive interferences between the two low-resolution images occur only if the wave-speed is correct, and maximum intensity and sharpness is observed in the envelope of the high-resolution image.

In this work, the autofocus method is applied to the composite image as defined by Equation 15. In fact, the autofocus method can be applied to a single-type image. For instance, we applied the autofocus method to PPPP images in [20] in order to estimate the parameters of a model of weak transverse isotropy. Here, we try to estimate the parameters of a model of exact transverse isotropy. The online supplementary document shows that the metrics of image quality applied to single-type images (PPPP, PSPP/PPSP or PSSP) provide non-convex objective functions, hence not suitable for solving the optimization problem. Thus the joint estimation of a pair of parameters (for instance $\delta^{*}$ and $\beta_{0}$ ) will be performed with the composite image, because it generates convex objective functions. 


\section{ESTIMATION OF THE COMPRESSIONAL AND SHEAR WAVE-SPEEDS IN A TUBE MADE OF MATERIAL WITH}

\section{ISOTROPIC ELASTICITY}

The approach is first evaluated for measuring the compressional and shear wave-speeds and the thickness of a tube of Polymethyl Methacrylate (PMMA), i.e. a material with isotropic elasticity. The outer and inner diameter of the tube was $20 \mathrm{~mm}$ and $8 \mathrm{~mm}$. Ultrasound data acquisitions in the transverse and longitudinal planes of the tube were repeated 10 times, with repositioning of the probe between each acquisition. Figure 6 shows the 4 different types of reconstructed images of the tube, and the composite image created as the sum of the 4 normalized images using the PPPP, PSPP, PPSP and PSSP paths.

Steps 1 to 4 a of our proposed strategy (see Section III-A) were applied to estimate jointly the compressional and shear wave-speeds, $V_{P}$ and $V_{S}$. PMMA is a material with isotropic elasticity, and hence we did not try to estimate $\beta_{0}$ and $\delta^{*}$ (step 4d). Instead we estimated $V_{P}$ and $V_{S}$ in transverse and longitudinal planes. If our approach is sound, the same values of $V_{P}$ and $V_{S}$ should be obtained in transverse and longitudinal planes of the PMMA tube. In addition, the compressional wave-speed was measured with the head-wave velocity (see Appendix B). Next, similar to the work reported in [20], the compressional and shear wave-speeds were also estimated separately, in PPPP images (longitudinal and transverse) and in PSSP images (longitudinal and transverse), respectively. Finally, steps $5 \mathrm{a}$ to $5 \mathrm{c}$ were applied for estimating the thickness of the tube.

Table I summarizes the results. The estimated compressional and shear wave-speeds are in good agreement with literature [41]. Joint and separate estimations of the compressional wave-speed with the autofocus method are in good agreement, in longitudinal and transverse images. And these estimates are close to the measured head-wave velocity. As for the shear wave-speed, joint and separate estimations with the autofocus method are in good agreement in longitudinal images. In transverse images, while the joint estimation was successful, we failed to estimate the shear wave-speed separately in PSSP images. This issue is discussed in Section VII-C. Once the compressional wavespeed was estimated, the thickness was measured in PPPP images (see Appendix C for details). The tube thickness is recovered with an error of less than $0.3 \%$.

It can be noticed in Figure 6 that PSPP, PPSP and PSSP images display two additional interfaces that are not located where the true inner interface is (cyan dash-dot line). PPPP, PSPP/PPSP and PSSP paths (see Figure 3) produce echo signals that do not overlap in the raw echo signals (Figure 9). The PPPP echo signal is recorded first, next the PSPP/PPSP echo signal and then the PSSP echo signal. In the approach used in this work, the different types of echo signals are not extracted from the raw echo signals before image reconstruction. During image reconstruction, a travel time is converted to a distance via the wave-speed. As a result, spurious interfaces appear in images (Figure 6). For instance, in a PSSP image, all echo signals being recorded later than the arrival time of the reflection at the outer surface are "migrated" using the shear wave-speed. While the PSSP echo signal is correctly "migrated" at the true position of the inner interface, the PPPP and PSPP/PPSP echo signals are not. Consequently two spurious interfaces appear in a PSSP image, corresponding to the PPPP and PSPP/PPSP paths. Even if the spurious interfaces make the interpretation of the images difficult, it does not significantly impact the 
exploitation of the composite image for estimating the parameters of the model of exact transverse isotropy. That is why we decided not to implement an additional step of signal processing for muting or extracting components of the raw echo signals.

This section shows that the joint estimation of the compressional and shear wave-speeds with the autofocus method applied to a composite image of a tube made of a material with isotropic elasticity was successful.

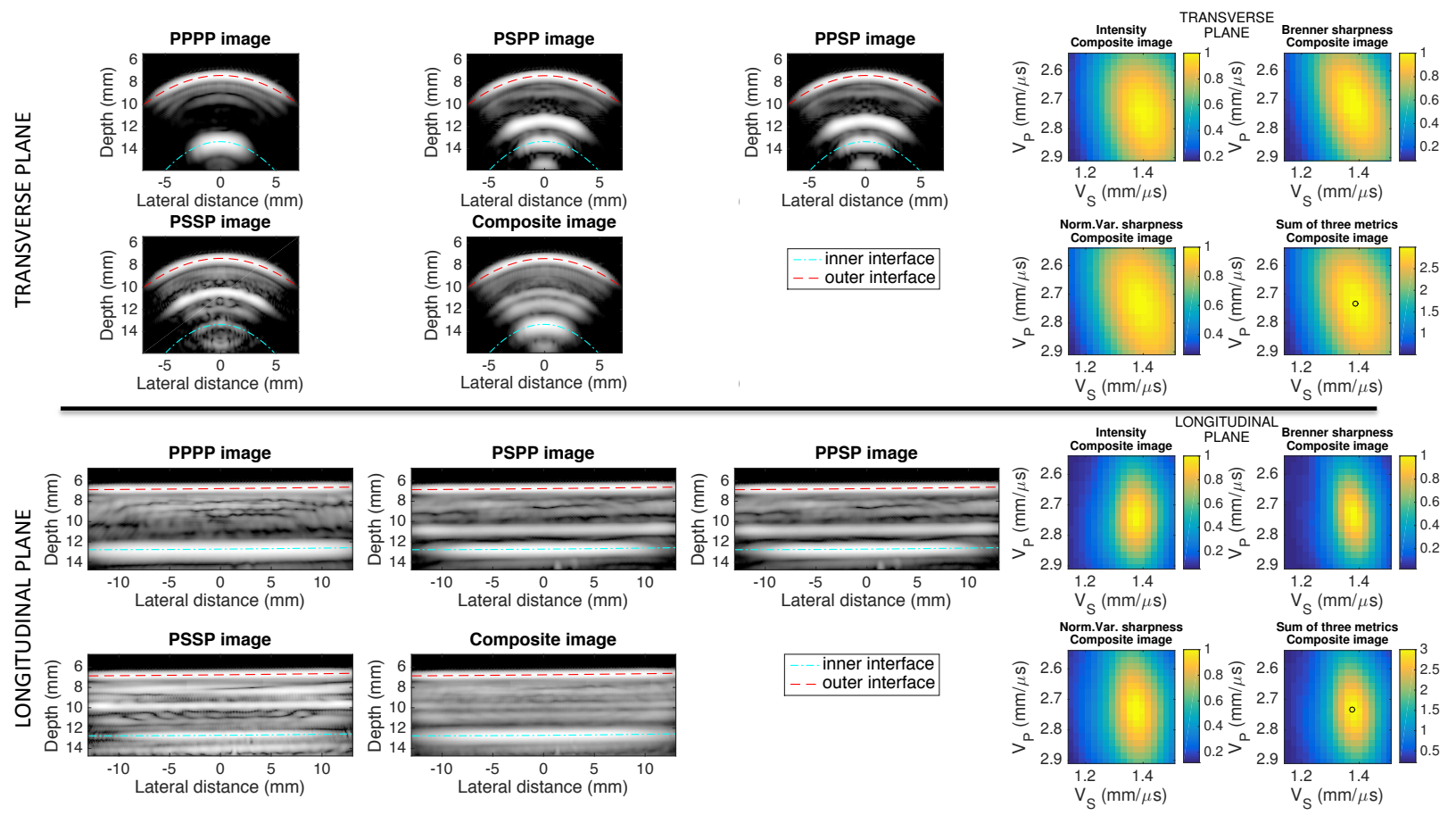

Fig. 6. Ultrasound images of the PMMA tube. Top: in a transverse plane. Bottom: in a longitudinal plane. A result of the autofocus method is shown on the right panels. Three metrics of image quality are investigated: image intensity, Brenner sharpness and normalized-variance sharpness. A circle shows the estimated values on the sum of three metrics. 


\begin{tabular}{|c|c|c|c|c|c|}
\hline & $\begin{array}{c}\text { ground truth or } \\
\text { comparison with literature }\end{array}$ & $\begin{array}{l}\text { ultrasound imaging } \\
\pm \text { precision }\end{array}$ & $\begin{array}{l}\text { acquisition } \\
\text { plane }\end{array}$ & $\begin{array}{l}\text { method and } \\
\text { image type }\end{array}$ & $\begin{array}{l}\text { error } \\
(\%)\end{array}$ \\
\hline thickness & $\begin{array}{c}6.0 \pm 0.1 \mathrm{~mm} \\
\text { (calliper ground truth } \\
\pm \text { uncertainty) }\end{array}$ & $\begin{array}{l}6.00 \pm 0.02 \mathrm{~mm} \\
5.99 \pm 0.03 \mathrm{~mm}\end{array}$ & $\begin{array}{l}\text { longitudinal } \\
\text { transverse }\end{array}$ & $\begin{array}{l}\text { PPPP image, isotropy } \\
\text { PPPP image, isotropy }\end{array}$ & $\begin{array}{l}0.1 \\
0.3\end{array}$ \\
\hline$V_{P}$ & $\begin{array}{l}\text { 2.69-2.75 mm/ } \mu \mathrm{s} \\
\quad \text { Ref. [41] }\end{array}$ & $\begin{array}{l}2.74 \pm 0.01 \mathrm{~mm} / \mu \mathrm{s} \\
2.76 \pm 0.01 \mathrm{~mm} / \mu \mathrm{s} \\
2.74 \pm 0.01 \mathrm{~mm} / \mu \mathrm{s} \\
2.78 \pm 0.02 \mathrm{~mm} / \mu \mathrm{s} \\
2.73 \pm 0.01 \mathrm{~mm} / \mu \mathrm{s}\end{array}$ & $\begin{array}{c}\text { longitudinal } \\
\text { longitudinal } \\
\text { longitudinal } \\
\text { transverse } \\
\text { transverse } \\
\end{array}$ & $\begin{array}{c}\text { headwave velocity } \\
\text { autofocus, PPPP image } \\
\text { autofocus, composite image } \\
\text { autofocus, PPPP image } \\
\text { autofocus, composite image }\end{array}$ & $\begin{array}{l}\text { NA } \\
\text { NA } \\
\text { NA } \\
\text { NA } \\
\text { NA }\end{array}$ \\
\hline$V_{S}$ & $\begin{array}{l}1.32-1.40 \mathrm{~mm} / \mu \mathrm{s} \\
\text { Ref. [41] }\end{array}$ & $\begin{array}{r}1.38 \pm 0.01 \mathrm{~mm} / \mu \mathrm{s} \\
1.38 \pm 0.01 \mathrm{~mm} / \mu \mathrm{s} \\
\mathrm{NA} \\
1.39 \pm 0.01 \mathrm{~mm} / \mu \mathrm{s} \\
\text { TABLE I }\end{array}$ & $\begin{array}{c}\text { longitudinal } \\
\text { longitudinal } \\
\text { transverse } \\
\text { transverse }\end{array}$ & $\begin{array}{c}\text { autofocus, PSSP image } \\
\text { autofocus, composite image } \\
\text { autofocus, PSSP image } \\
\text { autofocus, composite image }\end{array}$ & $\begin{array}{l}\text { NA } \\
\text { NA } \\
\text { NA } \\
\text { NA }\end{array}$ \\
\hline
\end{tabular}

ESTIMATED VALUES FOR THE TUBE OF PMMA.

\section{ESTIMATION OF THE PARAMETERS OF A MODEL OF EXACT TRANSVERSE ISOTROPY AND THE THICKNESS} OF A TUBE MADE OF A FIBER-REINFORCED COMPOSITE MATERIAL

We now address a more difficult problem: a tube made of a material with elastic anisotropy. We studied a tube made of a material that contains short glass fibers embedded in an epoxy matrix (Sawbones, Pacific Research Laboratories Inc., Vashon Island, WA, USA). It was designed to have elastic properties close to those of cortical bone. The elastic constants of the fiber-reinforced composite material were estimated with resonant ultrasound spectroscopy (RUS) measurements [42]. Parallelepipedic samples were extracted from 5mm-thick and 8mm-thick plates. From these estimates of the elastic stiffnesses and the mass density, we calculated the corresponding values of Thomsen's parameters (Table II). It was shown that a model of transverse isotropy describes well the elasticity of this material [42], [43]. We studied a tube with outer and inner diameters of $35 \mathrm{~mm}$ and $27 \mathrm{~mm}$, respectively. Like with the PMMA tube, ultrasound data acquisitions were repeated 10 times in the transverse and longitudinal planes of the tube, with repositioning of the probe between each acquisition.

The 4 different types of reconstructed images of the tube, and the composite image created as the sum of the 4 normalized images using the PPPP, PSPP, PPSP and PSSP paths are plotted in Figure 7. Figure 7 also shows how the quality metrics of the composite image evolve as functions of the tested values, $\delta^{*}$ and $\beta_{0}$ in a longitudinal plane, or $V_{P}^{\text {radial }}$ and $V_{S H}^{\text {radial }}$ in a transverse plane. Table III shows that the estimates obtained with the autofocus method are in good agreement with the values calculated from resonant ultrasound spectroscopy measurements reported in [42] (Table II). Both the weak-anisotropy and exact-anisotropy models provide a tube thickness in good agreement with the ground truth value obtained with a calliper (error $<0.3 \%$ ). Figure 7 shows that the angular dependence of the compressional, vertically- and horizontally-polarized shear wave-speeds (as estimated in this work) is in good agreement with that calculated from resonant ultrasound spectroscopy measurements reported in 


\begin{tabular}{c|c} 
Mass density & $1640 \pm 30 \mathrm{~kg} / \mathrm{m}^{3}$ \\
\hline$C_{11}$ & $14.75 \pm 0.7 \mathrm{GPa}$ \\
\hline$C_{33}$ & $22.8 \pm 0.9 \mathrm{GPa}$ \\
\hline$C_{13}$ & $7.85 \pm 0.85 \mathrm{GPa}$ \\
\hline$C_{44}$ & $4.36 \pm 0.025 \mathrm{GPa}$ \\
\hline$C_{66}$ & $3.72 \pm 0.03 \mathrm{GPa}$ \\
\hline$V_{P}^{\text {axial }}\left(\right.$ or $\left.\alpha_{0}\right)$ & $3.73 \pm 0.08 \mathrm{~mm} / \mu \mathrm{s}$ \\
\hline$\beta_{0}$ & $1.63 \pm 0.02 \mathrm{~mm} / \mu \mathrm{s}$ \\
\hline$\delta^{*}$ & $-0.22 \pm 0.05$ \\
\hline$V_{P}^{\text {radial }}$ & $3.00 \pm 0.08 \mathrm{~mm} / \mu \mathrm{s}$ \\
\hline$V_{S H}^{\text {radial }}$ & $1.51 \pm 0.02 \mathrm{~mm} / \mu \mathrm{s}$ \\
\hline$\epsilon$ & $-0.18 \pm 0.02$
\end{tabular}

MASS DENSITY, ELASTIC CONSTANTS AND THEIR UNCERTAINTY FOR THE FIBER-REINFORCED COMPOSITE MATERIAL MEASURED WITH RESONANT ULTRASOUND SPECTROSCOPY [42]. CORRESPONDING VALUES OF THOMSEN'S PARAMETERS ARE ALSO GIVEN.

[42] (Table II). Interestingly it was possible to estimate $V_{S H}^{\text {radial }}$ in composite transverse images, while a separate estimation in PSSP transverse images failed. This is further discussed in Section VII-C.

This section demonstrates that the proposed method applied to the composite image of a tube made of a material with anisotropic elasticity succeeded in estimating the 5 parameters of a model of exact transverse isotropy.

\begin{tabular}{|c|c|c|c|c|c|}
\hline & $\begin{array}{c}\text { ground truth or } \\
\text { comparison with literature }\end{array}$ & $\begin{array}{l}\text { ultrasound imaging } \\
\quad \pm \text { precision }\end{array}$ & $\begin{array}{l}\text { acquisition } \\
\text { plane }\end{array}$ & $\begin{array}{l}\text { method and } \\
\text { image type }\end{array}$ & $\begin{array}{l}\text { error } \\
(\%)\end{array}$ \\
\hline thickness & $\begin{array}{l}4.0 \pm 0.1 \mathrm{~mm} \\
\text { (calliper ground truth } \\
\pm \text { uncertainty) }\end{array}$ & $\begin{array}{l}4.01 \pm 0.02 \mathrm{~mm} \\
4.00 \pm 0.03 \mathrm{~mm} \\
3.99 \pm 0.02 \mathrm{~mm}\end{array}$ & $\begin{array}{c}\text { longitudinal } \\
\text { longitudinal } \\
\text { transverse }\end{array}$ & $\begin{array}{l}\text { PPPP image, exact anisotropy } \\
\text { PPPP image, weak anisotropy } \\
\text { PPPP image }\end{array}$ & $\begin{array}{l}0.3 \\
0.1 \\
0.3\end{array}$ \\
\hline$V_{P}^{\text {axial }}\left(\right.$ or $\left.\alpha_{0}\right)$ & $3.73 \pm 0.08 \mathrm{~mm} / \mu$ s (Table II) & $3.71 \pm 0.01 \mathrm{~mm} / \mu \mathrm{s}$ & longitudinal & headwave velocity & NA \\
\hline$V_{P}^{\text {radial }}$ & $3.00 \pm 0.08 \mathrm{~mm} / \mu \mathrm{s}$ (Table II) & $\begin{array}{l}3.05 \pm 0.03 \mathrm{~mm} / \mu \mathrm{s} \\
3.01 \pm 0.01 \mathrm{~mm} / \mu \mathrm{s}\end{array}$ & $\begin{array}{l}\text { transverse } \\
\text { transverse }\end{array}$ & $\begin{array}{c}\text { autofocus, PPPP image } \\
\text { autofocus, composite image }\end{array}$ & $\begin{array}{l}\text { NA } \\
\text { NA }\end{array}$ \\
\hline$V_{S H}^{\text {radial }}$ & $1.51 \pm 0.02 \mathrm{~mm} / \mu \mathrm{s}$ (Table II) & $\begin{array}{c}1.53 \pm 0.02 \mathrm{~mm} / \mu \mathrm{s} \\
\mathrm{NA}\end{array}$ & $\begin{array}{l}\text { transverse } \\
\text { transverse }\end{array}$ & $\begin{array}{c}\text { autofocus, composite image } \\
\text { autofocus, PSSP image }\end{array}$ & $\begin{array}{l}\text { NA } \\
\text { NA }\end{array}$ \\
\hline$\beta_{0}$ & $1.63 \pm 0.02 \mathrm{~mm} / \mu$ s (Table II) & $1.61 \pm 0.01 \mathrm{~mm} / \mu \mathrm{s}$ & longitudinal & autofocus, composite image, exact anisotropy & NA \\
\hline$\delta^{*}$ & $-0.22 \pm 0.05$ (Table II) & $-0.20 \pm 0.03$ & longitudinal & autofocus, composite image, exact anisotropy & NA \\
\hline$\epsilon$ & $-0.18 \pm 0.02$ (Table II) & $-0.16 \pm 0.01$ & NA & calculated from $V_{P}^{a x i a l}$ and $V_{P}^{\text {radial }}$ & NA \\
\hline$\xi$ & & $\begin{array}{r}2.02 \pm 0.04 \\
\text { TABLE III }\end{array}$ & longitudinal & autofocus, PPPP image, weak anisotropy & NA \\
\hline
\end{tabular}

ESTIMATED VALUES FOR THE TUBE OF FIBER-REINFORCED COMPOSITE MATERIAL. 

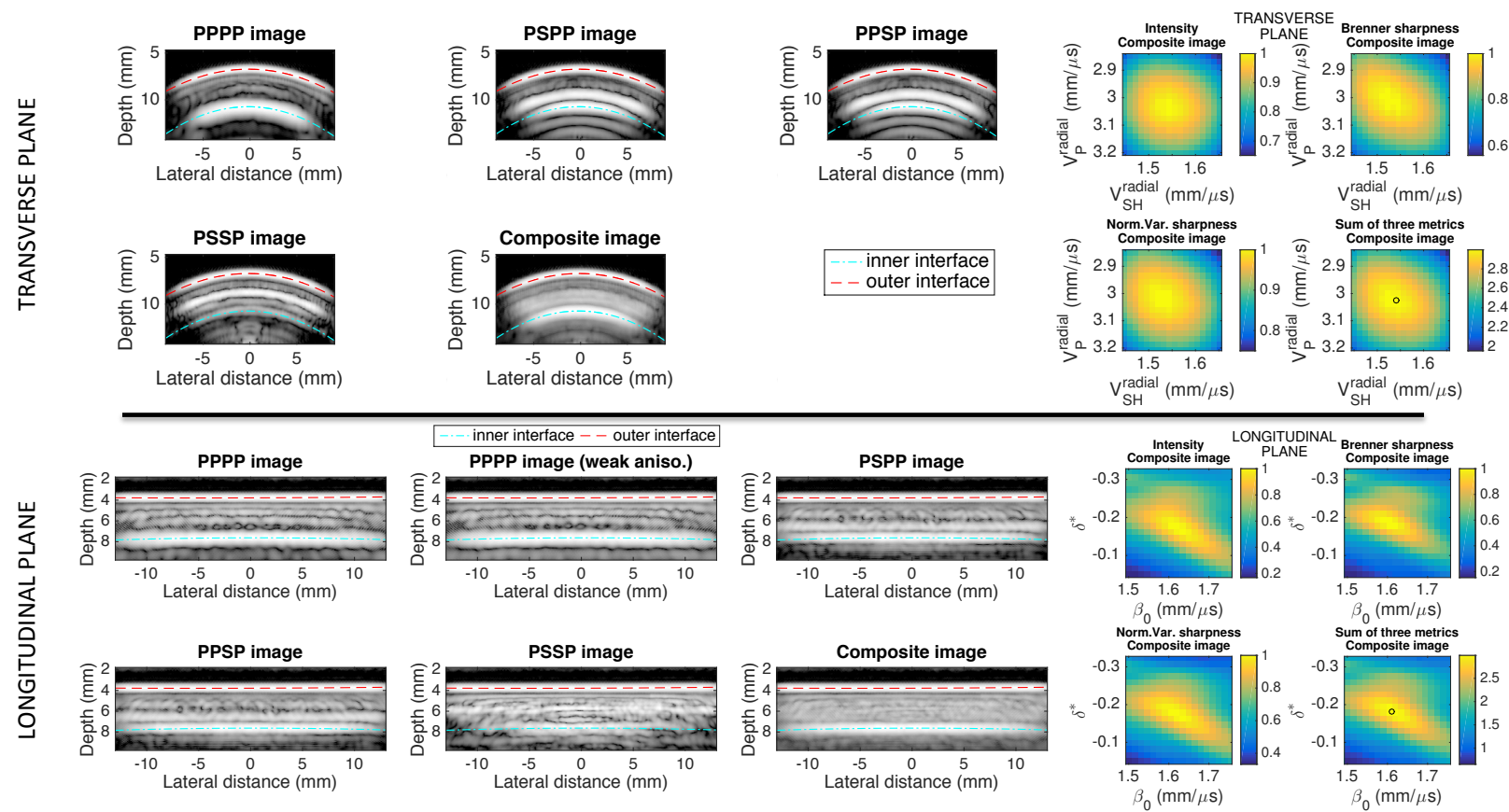

$\beta_{0}(\mathrm{~mm} / \mu \mathrm{S})$ Sum of three metrics
Composite image

\begin{tabular}{|c|}
\hline 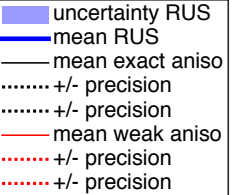 \\
\hline
\end{tabular}
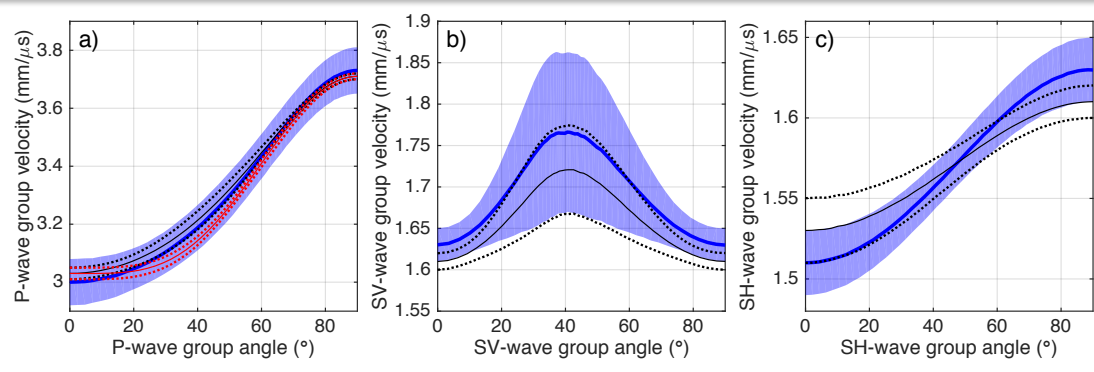

Fig. 7. Ultrasound images of the tube of fiber-reinforced composite material. Top: in a transverse plane. Middle: in a longitudinal plane. A result of the autofocus method is shown on the right panels. Three metrics of image quality are investigated: image intensity, Brenner sharpness and normalized-variance sharpness. A circle shows the estimated values on the sum of three metrics. Bottom: anisotropy of compressional wave-speed (panel a), vertically-polarized shear wave-speed (panel b) and horizontally-polarized shear wave-speed (panel c) in the fiber-reinforced composite material (Sawbones), calculated from the elastic constants measured with resonant ultrasound spectroscopy [42] (table II), and measured in this work with ultrasound imaging and the autofocus approach on a 4-mm thick tube.

\section{In vivo FEASIBILITY OF THE APPROACH AT THE RADIUS BONE}

Finally we evaluate the feasibility of the method in vivo in cortical bone. Ultrasound recordings were acquired at the left radius bone (forearm) of a healthy volunteer recruited from the laboratory staff. Informed consent of the 37 -year-old subject was obtained. The center of the ultrasound probe was placed $80 \mathrm{~mm}$ away from the distal end of the radius (wrist joint). The ground truth thickness of the cortex of the radius was obtained in a previous study (volunteer 1 in [20]) with site-matched high-resolution X-ray peripheral computed tomography (XtremeCT, Scanco Medical, Switzerland; voxel size $82 \mu \mathrm{m}$ ). All methods were performed in accordance with the relevant guidelines and regulations. 
Ultrasound data acquisitions in the transverse and longitudinal planes were repeated 10 times, with repositioning of the probe between each acquisition. Accurate positioning of the ultrasound probe with respect to the radius bone was achieved thanks to a real-time implementation of the work described in [20] which generated 4 images per second. With the autofocus method, we obtained a compressional wave-speed in cutaneous tissue of $c_{\text {tissue }}=1.65 \pm 40$ $\mathrm{mm} / \mu \mathrm{s}$, in good agreement with literature [44]. Figure 8 shows the 4 different types of reconstructed images of the cortex of the radius bone, and the composite image created as the sum of the 4 normalized images using the PPPP, PSPP, PPSP and PSSP paths. Figure 8 shows how the quality metrics of the composite image evolve as functions of the tested values of $\delta^{*}$ and $\beta_{0}$.

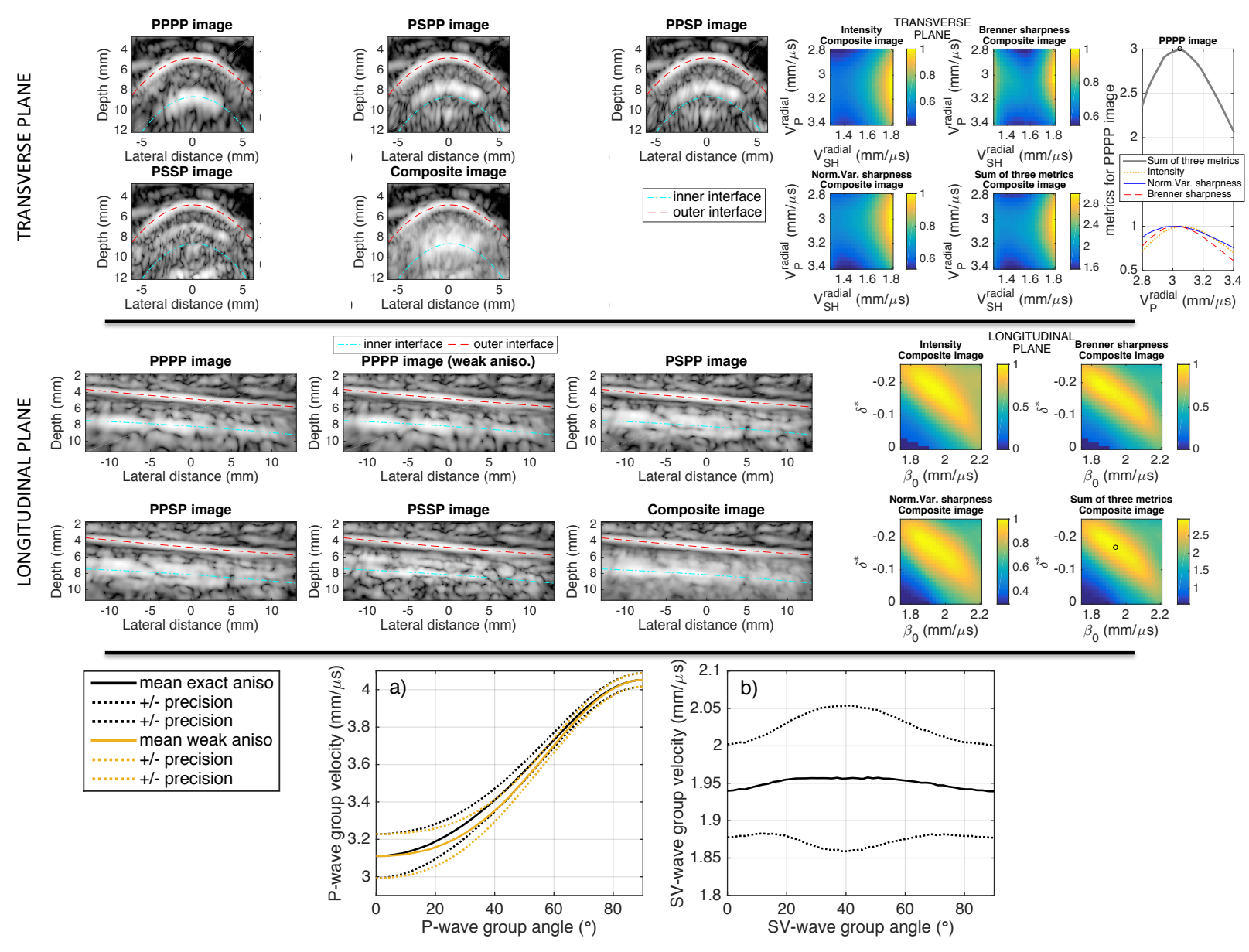

Fig. 8. Ultrasound images of the cortex of the radius bone (forearm) of a healthy volunteer. Top: in a transverse plane (with a priori value for $V_{S H}^{\text {radial }}=1.7 \mathrm{~mm} / \mu \mathrm{s}$ ). Middle: in a longitudinal plane. A result of the autofocus method is shown on the right panel. Three metrics of image quality are investigated: image intensity, Brenner sharpness and normalized-variance sharpness. It is seen that the method fails to estimate $V_{S H}^{\text {radial }}$ with the composite image in the transverse plane, $V_{P}^{\text {radial }}$ is estimated with transverse PPPP images. A circle shows the estimated values on the sum of three metrics. Bottom: anisotropy of compressional wave-speed (panel a) and vertically-polarized shear wave-speed (panel b) measured in vivo in cortical bone.

Interestingly the values of Thomsen's parameters estimated in vivo (Table IV) are in good agreement with the 


\begin{tabular}{|c|c|c|c|}
\hline & $\begin{array}{l}\text { ultrasound imaging } \\
\quad \pm \text { precision }\end{array}$ & $\begin{array}{l}\text { acquisition } \\
\text { plane }\end{array}$ & $\begin{array}{l}\text { method and } \\
\text { image type }\end{array}$ \\
\hline thickness & $\begin{array}{l}3.53 \pm 0.08 \mathrm{~mm} \\
3.56 \pm 0.11 \mathrm{~mm} \\
3.62 \pm 0.13 \mathrm{~mm}\end{array}$ & $\begin{array}{l}\text { longitudinal } \\
\text { longitudinal } \\
\text { transverse }\end{array}$ & $\begin{array}{l}\text { PPPP image, exact anisotropy } \\
\text { PPPP image, weak anisotropy } \\
\text { PPPP image }\end{array}$ \\
\hline$V_{P}^{a x i a l}$ & $4.05 \pm 0.04 \mathrm{~mm} / \mu \mathrm{s}$ & longitudinal & headwave velocity \\
\hline$V_{P}^{\text {radial }}$ & $3.11 \pm 0.12 \mathrm{~mm} / \mu \mathrm{s}$ & transverse & autofocus, PPPP image \\
\hline$\beta_{0}$ & $1.94 \pm 0.06 \mathrm{~mm} / \mu \mathrm{s}$ & longitudinal & autofocus, composite image, exact anisotropy \\
\hline$\delta^{*}$ & $-0.17 \pm 0.03$ & longitudinal & autofocus, composite image, exact anisotropy \\
\hline$\epsilon$ & $-0.20 \pm 0.02$ & NA & calculated from $V_{P}^{a x i a l}$ and $V_{P}^{\text {radial }}$ \\
\hline$\xi$ & $1.67 \pm 0.08$ & $\begin{array}{r}\text { longitudinal } \\
\text { TABL }\end{array}$ & $\begin{array}{l}\text { autofocus, PPPP image, weak anisotropy } \\
\text { IV }\end{array}$ \\
\hline
\end{tabular}

RESULTS OBTAINED In vivo AT THE RADIUS BONE (FOREARM) OF A HEALTHY VOLUNTEER.

distribution of values measured on ex vivo samples of human cortical bone with resonance ultrasound spectroscopy (Figure 1). A thickness of the cortical bone layer of $3.7 \pm 0.2 \mathrm{~mm}$ was obtained with high-resolution X-ray peripheral computed tomography [20]. The weak-anisotropy and exact-anisotropy models provide a thickness in the longitudinal view of $3.56 \pm 0.11 \mathrm{~mm}$ and $3.53 \pm 0.08 \mathrm{~mm}$, respectively. In the transverse view, the estimated thickness in the ultrasound image is $3.62 \pm 0.13 \mathrm{~mm}$. Thus the good agreement with high-resolution X-ray peripheral computed tomography indicates that the estimated velocity model is good. Figure 8 shows the dependence of the compressional and vertically-polarized shear wave-speeds on the group angle, as calculated with the parameters estimated in vivo. Unfortunately, we were not able to estimate $V_{S H}^{\text {radial }}$ with composite transverse images, possible reasons are addressed in Section VII-C.

These results show that the estimation of $\alpha_{0}, V_{P}^{\text {radial }}$ (or $\epsilon$ ), $\delta^{*}$ and $\beta_{0}$ seems feasible in vivo.

\section{DISCUSSION}

\section{A. Hypotheses, limitations and advantages of the proposed approach}

The hypotheses made by the proposed method are listed and discussed below.

- Each layer (cutaneous tissue and cortical bone) is assumed homogeneous at the mesoscopic scale (millimeter): assuming homogeneous layers holds for relatively young and healthy subjects, however this assumption would likely not hold in the elderly population or in subjects with severe osteoporosis because the bone cortex can become highly heterogeneous [45]

- The elasticity is assumed to be well described with a model of transverse isotropy: a large number of studies have shown that the elasticity of cortical bone at the diaphysis of a long bone is well described with transverse isotropy (or hexagonal symmetry) [30]-[32], [46]

- The direction of maximum compressional wave-speed is assumed to be aligned with the outer surface of the elastic layer (in a longitudinal image): in human long bones, osteons align nearly parallel to the axis of the 
diaphysis and to the outer surface [47]-[49], the direction of maximum compressional wave-speed coincides with the orientation of the osteons

- The ultrasound transducer array is assumed to be aligned with the direction of maximum compressional wavespeed during an acquisition in a longitudinal plane: thanks to real-time imaging of the bone cortex during acquisition (4 images per second), we anticipate that the positioning error is of the order of 1 degree

- The ultrasound transducer array is assumed to be perpendicular to the direction of maximum compressional wave-speed during an acquisition in a transverse plane: thanks to real-time imaging of the bone cortex during acquisition (4 images per second), we anticipate that the positioning error is of the order of 1 degree

- The outer surface of bone is assumed to be flat in a longitudinal plane for accurate measurement of $\alpha_{0}$ with the headwave velocity (step $4 \mathrm{~b}$ of the proposed procedure, see also Appendix B): the width of the transducer array is $28 \mathrm{~mm}$, Figures 8 and 11 show that the outer surface is locally nearly flat

A limitation of our strategy is the propagation of errors throughout the procedure. Because the unknowns (velocity parameters but also segmentation of interfaces) are sequentially estimated, an error made on the first estimates impacts the estimations of the next unknowns. For instance, we have investigated the influence of a large error on the wave-speed in the overlaying fluid layer when estimating $V_{P}^{\text {radial }}$ with an acquisition in a transverse plane. A 5\% underestimation (or overestimation) of the speed of sound in the overlaying fluid layer leads to an overestimation (or underestimation) of $V_{P}^{\text {radial }}$ by up to $10 \%$. Nonetheless, despite this limitation and the hypotheses made by the method, the thickness of the two tubes was measured with an error smaller than $0.3 \%$ (sections IV and V). And the thickness of the cortex of a radius bone estimated in vivo was in good agreement with high-resolution X-ray peripheral computed tomography (section VI). Moreover the estimated parameters of the model of exact transverse anisotropy in the fiber-reinforced composite material are in good agreement with resonant ultrasound spectroscopy measurements (Table III).

Measuring the velocity and anisotropy of compressional and vertically-polarized shear waves was attempted in a longitudinal plane of the same fiber-reinforced composite material with guided waves and a dedicated ultrasound transducer array by Foiret et al. [43]. Their approach requires the joint estimation of five unknowns (thickness, $\beta_{0}$, and 3 ratios of elastic stiffnesses), which is a difficult optimization problem. Instead, our strategy exploits bulk waves and a commercial clinical ultrasound probe. The advantage of using bulk waves is that the 5 unknowns (thickness, $\alpha_{0}, V_{P}^{\text {radial }}$ or $\epsilon, \delta^{*}$ and $\beta_{0}$ ) are estimated via 4 separate problems (see Section III-A). $V_{P}^{\text {radial }}$ is first measured, then $\alpha_{0}$ is estimated, next $\delta^{*}$ and $\beta_{0}$ are jointly searched, and finally the thickness is measured in a PPPP image. In this respect, our approach is technically simpler to implement.

Minonzio et al. estimated in vivo the thickness and the porosity of the cortex of the radius bone with guided waves and a dedicated ultrasound transducer array [50]. Their approach assumed the elastic constants of the bone matrix (i.e. the elasticity of the mineralized collagen matrix at the microscopic scale) to be known, and porosity is used as a "scaling" parameter to calculate the stiffness coefficients at the mesoscopic scale (asymptotic homogenization was 
used assuming cortical bone contains cylindrical pores). In contrast, our strategy does not need prior knowledge of the elastic stiffnesses of the bone matrix, it characterizes the elasticity of cortical bone at the mesoscopic scale directly.

\section{B. Comparison of weak-anisotropy and exact transverse isotropy models for PPPP images and goodness of fit}

In this section, we propose to appreciate the goodness of fit by overlaying the arrival times of specular reflections (calculated with the estimated model parameters) on the recorded raw echo signals. Next we discuss the results obtained with weak-anisotropy and exact transverse isotropy models for PPPP images.

First, it is worth noticing that the method used for image reconstruction (delay-and-sum algorithm, see Appendix A) is a point-diffractor (or scatter-point) imaging technique, while we are in fact interested in specular reflections by interfaces. However, since an interface can be approximated as a large number of point-diffractors next to each other, the technique performs well with specular reflections. As a matter of fact, it is extensively used by seismologists whose goal is to image interfaces between the many layers of geomaterials in the subsurface of the Earth [51]. Here, we show a post hoc verification of the model fitting. Once the velocity model was determined and after image reconstruction and segmentation of the two interfaces, it is possible to calculate the arrival time of specular reflections. Figures 9 and 9 show the envelope of raw echo signals acquired in the PMMA tube, the tube made of a fiber-reinforced composite material and in vivo at the radius bone. The arrival times of the four types of specular reflections exploited for image reconstruction (PPPP, PSSP, PSPP and PPSP) were calculated for a given transmit element of the transducer array.

For acquisitions in a longitudinal plane (figure 9), the arrival time of the head-wave (see Appendix B) is also indicated. It is seen that, for acquisitions in both longitudinal and transverse planes, the predicted arrival times of specular reflections fit well the raw echo signals, since one expects the predicted times to be collocated with the crest of the signal envelope (see Appendix A).

We observed that weak-anisotropy and exact transverse isotropy models recovered the thickness correctly in PPPP images, both in the tube made of a fiber-reinforced composite material (see Table III) and in vivo in the cortex of the radius bone of a healthy volunteer (see Table IV). Therefore the model of exact transverse isotropy (Equation 1) does not improve the measurement of thickness, as compared to the model of weak transverse isotropy (Equation 12). Figure 9 shows that the models of exact and weak transverse isotropy lead to very close arrival times of the PPPP ray path. The difference is less than $50 \mathrm{~ns}$, it represents about $10 \%$ of the ultrasound period which explains why the two models provide virtually identical PPPP images (Figures 7 and 8). Thus, it is preferable to use the model of weak transverse isotropy for anatomical imaging because it reduces computational time during image reconstruction. On the other hand, if the goal is material characterization, then the model of exact transverse isotropy is more suitable because the elastic anisotropy of cortical bone is moderate, not weak (Section II). 

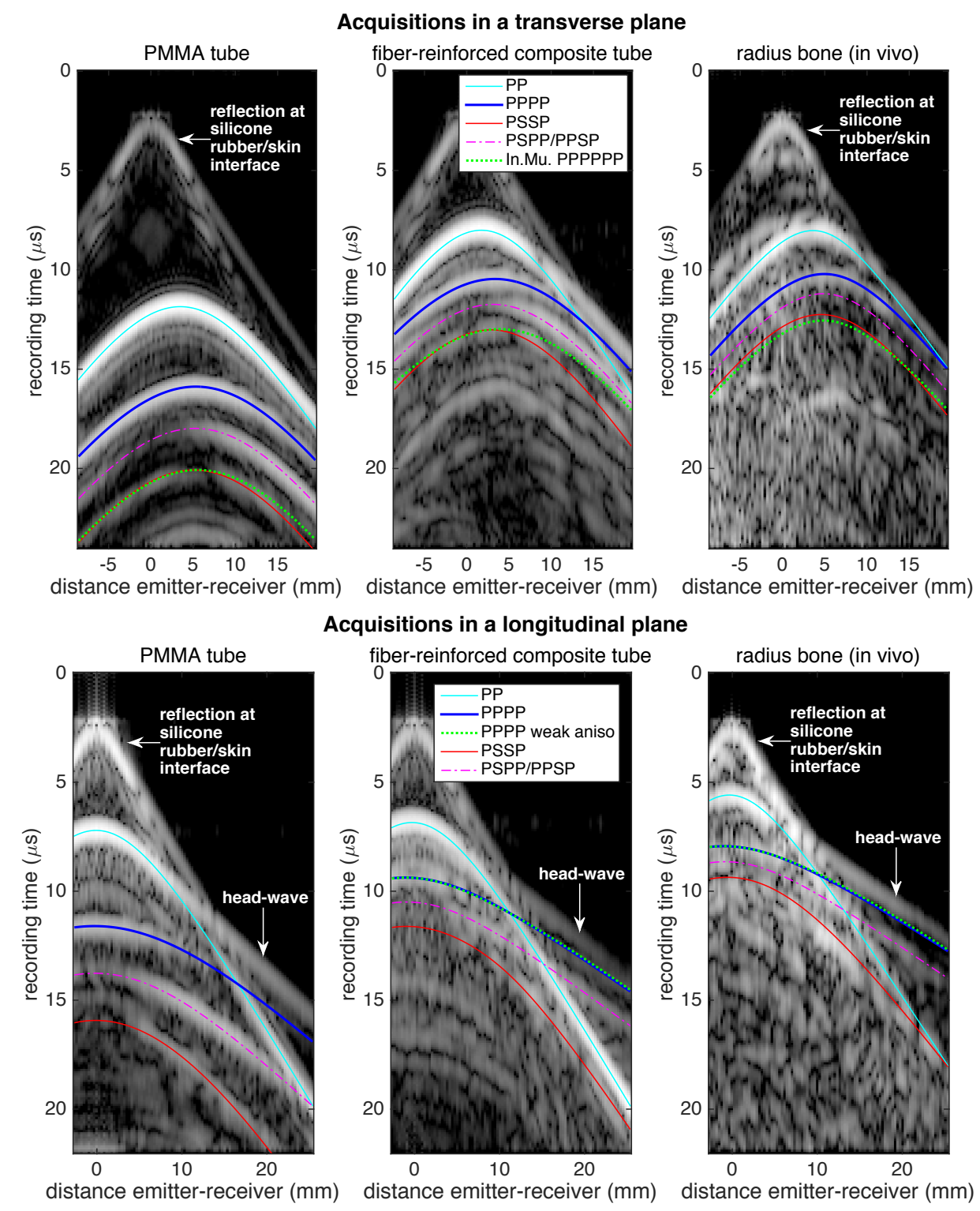

Fig. 9. Raw echo signals acquired in the PMMA tube, in the fiber-reinforced composite tube and in vivo at the radius bone (forearm). Top: in a transverse plane after the element 30 of the probe array has transmitted a short ultrasound burst. Bottom: in a longitudinal plane after the element 10 of the probe array has transmitted a short ultrasound burst. The log-compressed envelope of the echo signals is displayed with a dynamic range of $40 \mathrm{~dB}$, after time gain compensation. The theoretical arrival times of PP, PPPP, PSSP and PSPP/PPSP paths are calculated with the values of the parameters as estimated with the proposed method. In transverse planes (top panels), the arrival time of the internal multiple reflection within the elastic layer for a PPPPPP ray is depicted with a dotted green line (with a priori value for $V_{S H}^{\text {radial }}=1.7 \mathrm{~mm} / \mu \mathrm{s}$ for the radius bone). In longitudinal planes (bottom panels), the arrival time of a PPPP ray with the weak-anisotropy model is depicted with a dotted green line. Head-wave and reflection at the silicone rubber/skin interface are indicated with a white arrow.

\section{Measuring the velocity of a horizontally-polarized shear wave with an acquisition in a transverse plane}

The autofocus method applied to PSSP images failed to estimate $V_{S H}^{\text {radial }}$ (sections IV, V and VI). Although it succeeded with composite images in the tubes of PMMA and fiber-reinforced composite material, it failed in vivo 
at the radius bone. We believe the estimation of $V_{S H}^{\text {radial }}$ is very challenging for two reasons:

- The coefficient of transmission through the interface between the overlaying fluid layer and the elastic layer takes low values if the curvature of the elastic layer is large

- If diffuse scattering is strong like in vivo, the amplitude of a PSSP reflection echo can be smaller than that of diffuse scattering echoes

- The arrival time of a SH wave is close to that of a double internal reflection of P-wave if the curvature of the elastic layer is large

It is possible to calculate the relative power received by the elements of the transducer array by multiplying three coefficients for a plane wave (see for instance Chapter 2 in [52]):

- The forward coefficient of transmission through the interface between the overlaying fluid layer and the elastic layer, a mode conversion $\mathrm{P} \rightarrow \mathrm{S}$ occurs

- The coefficient of reflection of the shear wave at the interface between the underlaying fluid layer and the elastic layer

- The return coefficient of transmission through the interface between the overlaying fluid layer and the elastic layer, a mode conversion $\mathrm{S} \rightarrow \mathrm{P}$ occurs

As shown in Figure 10, using parameter values corresponding to a transverse acquisition in vivo, the relative received power calculated for a curved elastic layer is up to two orders of magnitude smaller than that calculated for a flat elastic layer. In the flat layer configuration, the incident angle at the interface can reach values up to 50 degrees (in absolute value), which leads to forward and return coefficients of transmission larger than 0.8 (Figure 10). In contrast, for the curved layer configuration, the incident angle at the interface does not exceed 18 degrees (in absolute value), resulting in forward and return coefficients of transmission smaller than 0.1 .

In fact, very similar numbers are obtained for an acquisition in a transverse plane of the PMMA tube, nonetheless the PSSP signal is recognizable in the raw echo signals (Figure 9). Unlike in vivo at the radius bone, diffuse scattering is much weaker in PMMA, this explains why the PSSP signal can be exploited in a transverse plane of the PMMA tube. The same calculations for PPPP rays show that the curvature of the elastic layer has a small influence on the received power. As for PSPP rays, the curvature of the elastic layer reduces the received power by a factor 10 , hence its effect is less than for PSSP rays.

At last, the arrival time of a SH-wave for an acquisition in a transverse plane is close to that of a double internal reflection of a P-wave (Figure 9). This is caused by the curvature of the elastic layer and by the fact that the compressional wave-speed is nearly twice larger than of the SH wave-speed. As a result the signals nearly overlap and hinder the estimation of $V_{S H}^{\text {radial }}$ with the autofocus method.

Possible improvements for the estimation of $V_{S H}^{\text {radial }}$ in vivo in cortical bone are the removal of internal multiples with techniques developed by seismologists (see for instance [53]) and the use of a lower ultrasound frequency in order to reduce the relative magnitude of diffuse scattering. 

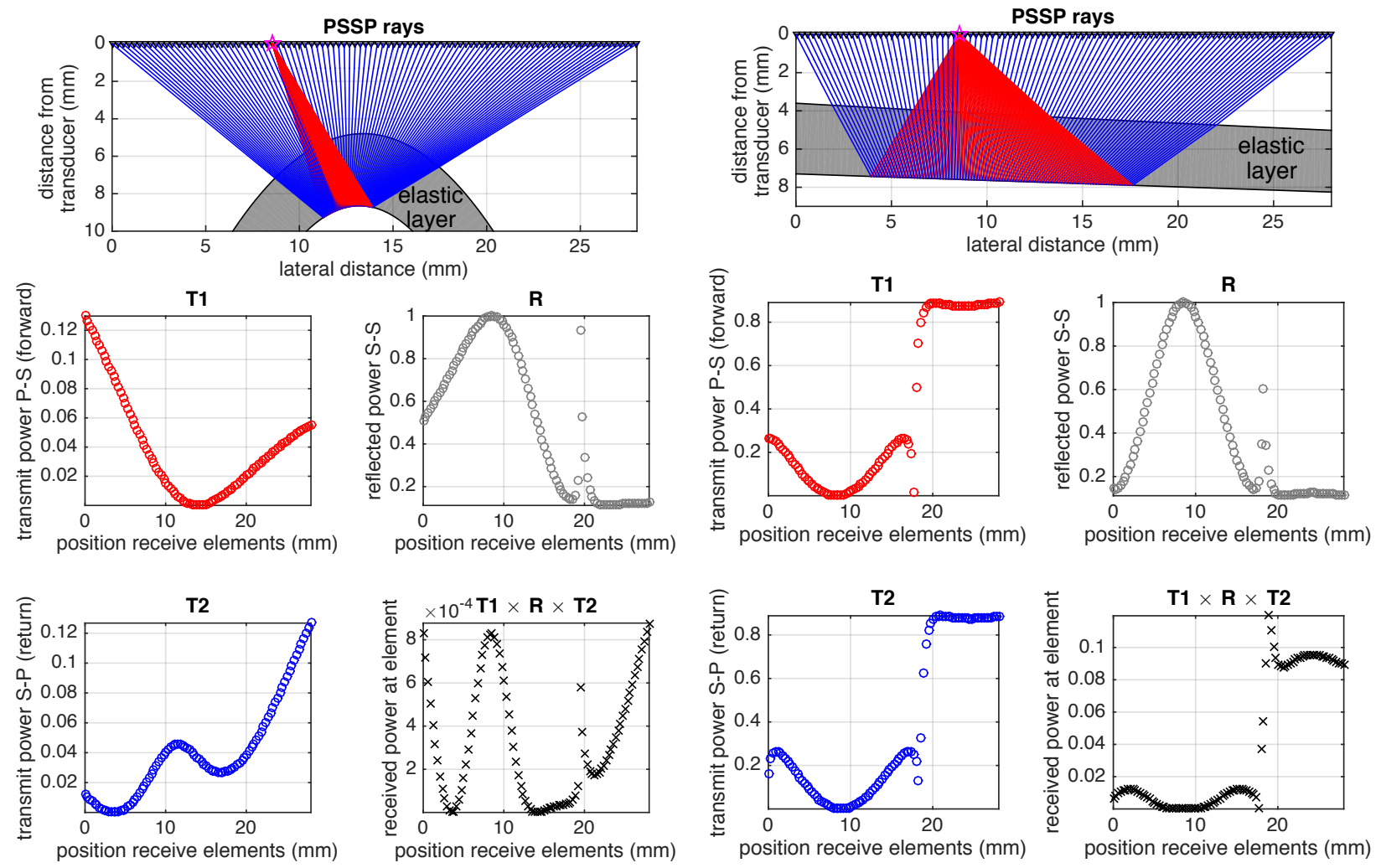

Fig. 10. Calculation of the relative power of a plane wave received by the elements of the transducer array for PSSP ray paths, for a curved elastic layer (left panels) and for a flat elastic layer (right panels), and for a given transmit element (depicted with a star). The elasticity of the layer is isotropic, with compressional and shear wave-speeds of $3.2 \mathrm{~mm} / \mu \mathrm{s}$ and $1.7 \mathrm{~mm} / \mu \mathrm{s}$, respectively. The compressional wave-speed in the overlaying and underlaying fluid layers is $1.65 \mathrm{~mm} / \mu \mathrm{s}$. The mass density for the elastic layer and the fluid layers is $1950 \mathrm{~kg} / \mathrm{m}^{3}$ and 1000 $\mathrm{kg} / \mathrm{m}^{3}$, respectively. Forward rays are plotted in blue, while return rays are red.

\section{CONCLUSION}

We demonstrated that the proposed method was able to estimate the 5 parameters of the model of exact transverse isotropy when applied to a tube made of bone-mimicking material. The estimates of the parameters were in good agreement with those calculated from resonant ultrasound spectroscopy measurements. The tube thickness was recovered with an error smaller than $0.3 \%$. The results obtained in vivo at the radius bone were promising, 4 parameters could be estimated and the estimates were in good agreement with ex vivo resonant ultrasound spectroscopy measurements reported in the literature. Moreover the thickness of the cortical bone layer in the ultrasound image was in good agreement with site-matched high-resolution X-ray peripheral computed tomography. These results were obtained on a single subject, they suggest that the approach is feasible in vivo. Finally, weakanisotropy and exact transverse isotropy models provided very close measurements of the thickness in the tube and in vivo at the radius bone. In consequence, we recommend using the model of weak transverse isotropy for anatomical imaging because it reduces computational time during image reconstruction. For material characterization however, the model of exact transverse isotropy is preferred because the elastic anisotropy of cortical bone is moderate, not 
weak.

\section{APPENDIX A}

\section{IMAGE RECONSTRUCTION}

Image reconstruction is based on the delay-and-sum algorithm (also called Kirchhoff migration in seismic imaging, see for instance [54], or Total focusing method in non-destructive testing, see for instance [55]). Travel times can be calculated with two-point ray tracing using Fermat's principle with Brent's method (see Chapter 10 in [39]) or by solving the eikonal equation (see for instance [54], [56]). Each image pixel is considered as a hypothetical scattering point. The reconstruction algorithm sums over the receive aperture of the probe array the recorded echo signals along the calculated round-trip travel times, and over the transmissions (coherent compounding):

$$
\begin{aligned}
\operatorname{Im}(x, z) & =\sum_{i T=1}^{N_{T}} \sum_{i R=1}^{N_{R}} W(x, z, i T) \times W(x, z, i R) \times D\left[t=t_{T}(i T, x, z)+t_{R}(i R, x, z), i T, i R\right] \\
W(x, z, k) & =\cos \left(\theta_{\text {ray angle at element }}(x, z, k)\right) \times \operatorname{sinc}\left(\sin \left(\theta_{\text {ray angle at element }}(x, z, k)\right) a / \lambda\right) / r(x, z, k)
\end{aligned}
$$

$x$ and $z$ are the cartesian coordinates of the pixel in the image. $N_{T}$ and $N_{R}$ are the number of transmit elements and the number of receive elements, respectively. In this work, $N_{T}=N_{R}=96 . W(x, z, k)$ is a weighting function, $k$ is the index of the (transmit or receive) element. The term $\operatorname{sinc}\left(\sin \left(\theta_{\text {ray angle at element }}(x, z, k)\right) a / \lambda\right)$ accounts for the directivity of an element. $a$ is the width of a single element of the probe array. $\lambda$ is the wavelength in cutaneous tissue (or water for measurements with a tube) at the center ultrasound frequency. $r(x, z, k)$ is the length of the ray from a transmit or receive element $k$ to a pixel in the image with coordinates $(x, z)$. $\theta_{\text {ray angle at element }}(x, z, k)$ is the ray angle at an element for a transmit or receive element $k$ and a pixel in the image with coordinates $(x, z) \cdot \cos \left(\theta_{\text {ray angle at element }}(x, z, k)\right)$ and $1 / r(x, z, k)$ are the obliquity factor and the spherical spreading factor, respectively (see for instance [54]). Note that the travel times, the ray angle at an element and the length of the ray are all output by the two-point ray tracing method. A band-pass filter with cut-off frequencies [1MHz $4 \mathrm{MHz}]$ is applied to the raw echo signals before image reconstruction. This operation reduces high-frequency noise and suppresses the DC offset.

Four different types of images were built in this work. Indeed an image can be reconstructed using the echo signals related either to the PPPP path, or to the PSSP path, or to the PSPP/PPSP paths (see Figure 3). For a given type of image, the corresponding outward travel time $t_{T}(i T, x, z)$ and return travel time $t_{R}(i R, x, z)$ are calculated. A medical ultrasound transducer contains a front layer made of a silicone rubber material. In most applications of medical ultrasound imaging, the effect of this layer on ultrasound propagation is corrected by a simple time delay during image reconstruction, refraction is not corrected because its effect is small. In this work however, the refraction caused by the silicone rubber layer was taken into account, the layer thickness is $1.3 \mathrm{~mm}$ and the speed of sound is $0.97 \mathrm{~mm} / \mu \mathrm{s}$. During calculation of travel times, the silicone rubber layer was included in the model, between the elements of the array and the cutaneous tissue (or water for measurements with a tube). 
Also, the delay-and-sum algorithm ignores the temporal duration of the transmit waveform. Optimal image reconstruction is achieved if the time lag between the very beginning of the received waveform (backscattered by a single target) and the time at which the envelope of the received waveform peaks is added to the travel times (or if the raw echo signals are shifted backwards in time by this time lag).

\section{APPENDIX B}

\section{ESTIMATION OF $\alpha_{0}$ WITH THE VELOCITY OF THE HEAD-WAVE}

Because the compressional wave-speed in the elastic layer is larger than that in the overlaying fluid layer, a head-wave (or lateral wave, see for instance [57]) is generated if the incident angle at the interface exceeds the critical angle $\arcsin \left(V_{\text {slow layer }} / V_{\text {fast layer }}\right)$. The head-wave propagates along the interface between the two layers with the velocity of a compressional wave in the elastic layer [58], [59]. For the fiber-reinforced composite tube and cortical bone in vivo, the velocity of the head-wave equals $\alpha_{0}$ (or $V_{P}^{a x i a l}$ ). If the ultrasound wavelength in the elastic layer (in this work less than $2 \mathrm{~mm}$ ) is smaller than the thickness of the elastic layer, and for a sufficient distance between the emitter and the receiver, the first recorded echo signal recorded by the probe array is the head-wave. The signal processing and calculation of the head-wave velocity is straightforward if the interface is flat [60]. As seen in Figure 9, the head-wave signal appears as a straight feature, its slope is related to the head-wave velocity. Because the interface is not parallel to the probe array, two sets of recorded signals are required: 1) when the first element of the array is the source and 2) when the last element of the array is the source. The apparent velocities of the head-wave $V_{1}$ and $V_{2}$ measured from these two configurations were estimated with the semblance method used by seismologists [61]. It is defined as:

$$
S=\frac{\sum_{n=N-M}^{N+M}\left(\sum_{i R=1}^{N_{R}} D(n, i T, i R)\right)^{2}}{N_{R} \sum_{n=N-M}^{N+M} \sum_{i R=1}^{N_{R}} D^{2}(n, i T, i R)}
$$

where $n$ and $N$ are time sample indices, $2 M+1$ is the length of the window along the time axis, $i T$ and $i R$ are the emitter and receiver indices, $D(n, i T, i R)$ is the signal amplitude at time index $n$. The semblance measures the coherence along the wavefront of the head-wave for a given test value of the head-wave velocity. The semblance is maximum if the test value of the head-wave velocity is correct. $\alpha_{0}$ is then equal to $\left(2 V_{1} V_{2} \cos (\psi)\right) /\left(V_{1}+V_{2}\right)[60]$. $\psi$ is the angle of the interface ( $\psi=0$ means that the interface is parallel to the probe array). The interface angle $\psi$ is measured in the ultrasound image. Before computing the semblance, a muting mask is designed using apriori maximum and minimum values of the head-wave velocity and applied to raw echo signals. Figure 11 illustrates the procedure for calculating the headwave velocity with signals acquired in vivo at the radius bone.

\section{APPENDIX C}

\section{MEASURING THE THICKNESS OF THE ELASTIC LAYER IN THE ULTRASOUND IMAGE}

Because images of the Sawbones plate and the bone cortex using the PPPP path exhibit the best contrast (better image quality than images exploiting PSSP and PSPP/PPSP paths), the thickness of the elastic layer was only assessed in P-wave based images. The segmentation method based on Dijkstra's algorithm [62], [63] seeks the 

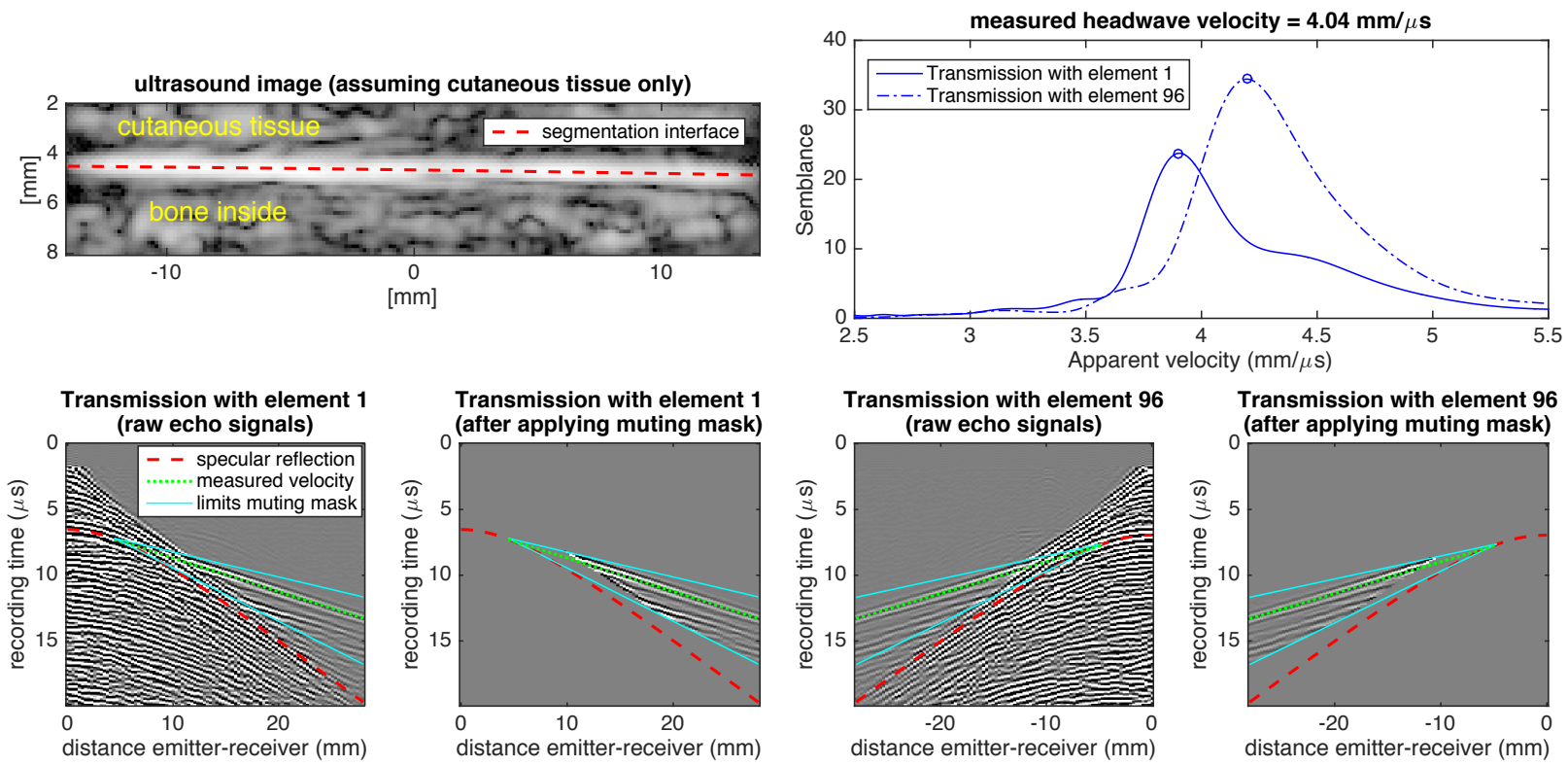

Fig. 11. Illustration of the procedure for calculating the headwave velocity with signals acquired in vivo at the radius bone. Top left: ultrasound image of the cutaneous tissue for segmentation of the outer surface of the bone and measurement of angle $\psi$. Bottom: raw echo signals acquired when the first (or last) element of the array is the source, the signals are shown before and after applying the muting mask. Top right: the semblance metrics calculated for multiple values of the apparent velocity between 2.5 and $5.5 \mathrm{~mm} / \mu \mathrm{s}$.

shortest path that follows the interface with highest intensity in the ultrasound image. Once the two interfaces have been segmented, they are approximated by two parabolas. Then the mean parabola is calculated. Next, about 50 line segments are computed with a direction normal to the mean parabola and bounded by the parabolas of the two interfaces. The thickness is estimated as the mean length of these line segments. This method was applied to transverse and longitudinal images.

\section{ACKNOWLEDGMENT}

This work has been funded by a French ANR program (17-CE19-0008-01).

\section{REFERENCES}

[1] J. F. Greenleaf, S. A. Johnson, and A. H. Lent, "Measurement of spatial distribution of refractive index in tissues by ultrasonic computer assisted tomography," Ultrasound in Medicine and Biology, vol. 3, no. 4, pp. 327-335, 1978.

[2] J. F. Greenleaf and R. C. Bahn, "Clinical imaging with transmissive ultrasonic computerized tomography," IEEE Transactions on Biomedical Engineering, vol. BME-28, no. 2, pp. 177-185, 1981.

[3] M. Sak, N. Duric, P. Littrup, L. Bey-Knight, H. Ali, P. Vallieres, M. E. Sherman, and G. L. Gierach, "Using speed of sound imaging to characterize breast density," Ultrasound in Medicine \& Biology, vol. 43, no. 1, pp. 91-103, 2017.

[4] J. Wiskin, B. Malik, R. Natesan, and M. Lenox, "Quantitative assessment of breast density using transmission ultrasound tomography," Med Phys, vol. 46, no. 6, pp. 2610-2620, 2019.

[5] M. E. Anderson and G. E. Trahey, "The direct estimation of sound speed using pulse-echo ultrasound," The Journal of the Acoustical Society of America, vol. 104, no. 5, pp. 3099-3106, 1998. 

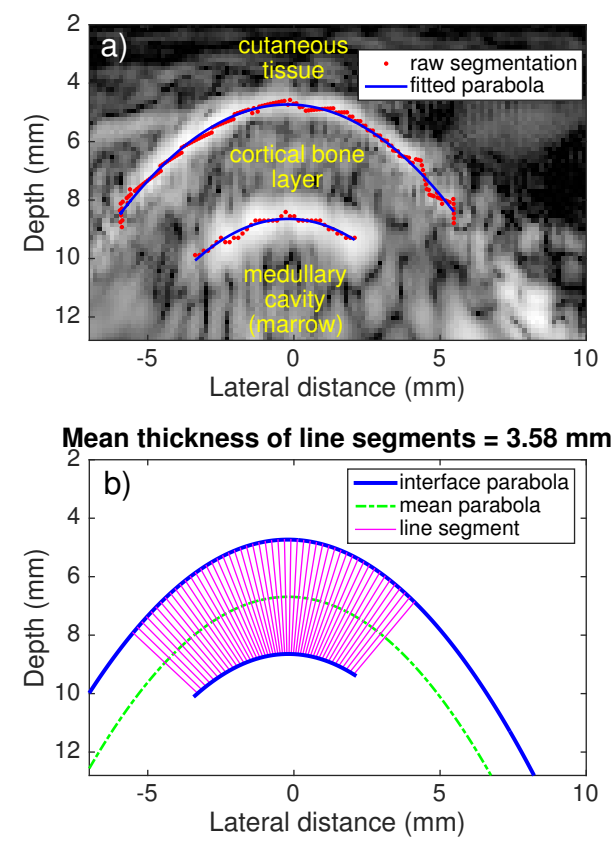

Fig. 12. Illustration of the method for measuring the mean thickness of the elastic layer in the ultrasound image. a) Segmentation of the two interfaces and fitting with a parabola in a transverse image of the radius bone. b) Calculation of mean thickness by computing multiple line segments.

[6] D. Napolitano, C. Chou, G. McLaughlin, T. Ji, L. Mo, D. DeBusschere, and R. Steins, "Sound speed correction in ultrasound imaging," Ultrasonics, vol. 44, Supplement, pp. e43 - e46, 2006.

[7] M. Imbault, A. Faccinetto, B.-F. Osmanski, A. Tissier, T. Deffieux, J.-L. Gennisson, V. Vilgrain, and M. Tanter, "Robust sound speed estimation for ultrasound-based hepatic steatosis assessment," Physics in Medicine \& Biology, vol. 62, no. 9, pp. 3582-3598, 2017.

[8] M. Imbault, M. D. Burgio, A. Faccinetto, M. Ronot, H. Bendjador, T. Deffieux, E. O. Triquet, P.-E. Rautou, L. Castera, J.-L. Gennisson, V. Vilgrain, and M. Tanter, "Ultrasonic fat fraction quantification using in vivo adaptive sound speed estimation," Physics in Medicine \& Biology, vol. 63, no. 21, p. 215013, 2018.

[9] H. Minh, J. Du, and K. Raum, "Estimation of thickness and speed of sound in cortical bone using multi-focus pulse-echo ultrasound," IEEE Trans Ultrason Ferroelectr Freq Control, vol. in press, 2019.

[10] M. Jaeger, E. Robinson, H. G. Akarcay, and M. Frenz, "Full correction for spatially distributed speed-of-sound in echo ultrasound based on measuring aberration delays via transmit beam steering," Physics in Medicine \& Biology, vol. 60, no. 11, pp. 4497-4515, 2015.

[11] S. J. Sanabria, E. Ozkan, M. Rominger, and O. Goksel, "Spatial domain reconstruction for imaging speed-of-sound with pulse-echo ultrasound: simulation and in vivo study," Physics in Medicine \& Biology, vol. 63, no. 21, p. 215015, 2018.

[12] M. Jakovljevic, S. Hsieh, R. Ali, G. Chau Loo Kung, D. Hyun, and J. J. Dahl, "Local speed of sound estimation in tissue using pulse-echo ultrasound: Model-based approach,” The Journal of the Acoustical Society of America, vol. 144, no. 1, pp. 254-266, 2018.

[13] W. Lambert, L. A. Cobus, M. Couade, M. Fink, and A. Aubry, "Reflection matrix approach for quantitative imaging of scattering media," Physical Review X, vol. 10, no. 2, pp. 021048-, 2020.

[14] J.-L. Gennisson, T. Deffieux, M. Fink, and M. Tanter, "Ultrasound elastography: Principles and techniques," Diagnostic and Interventional Imaging, vol. 94, no. 5, pp. 487-495, 2013.

[15] R. M. S. Sigrist, J. Liau, A. E. Kaffas, M. C. Chammas, and J. K. Willmann, "Ultrasound elastography: Review of techniques and clinical applications." Theranostics, vol. 7, no. 5, pp. 1303-1329, 2017.

[16] M. A. Greenfield, J. D. Craven, D. S. Wishko, A. L. Huddleston, R. Friedman, and R. Stern, "The modulus of elasticity of human cortical 
bone: An in vivo measurement and its clinical implications," Radiology, vol. 115, no. 1, pp. 163-166, 1975.

[17] M. A. Greenfield, J. D. Craven, A. Huddleston, M. L. Kehrer, D. Wishko, and R. Stern, "Measurement of the velocity of ultrasound in human cortical bone in vivo. estimation of its potential value in the diagnosis of osteoporosis and metabolic bone disease." Radiology, vol. 138, no. 3, pp. 701-710, 1981.

[18] M. Daugschies, K. Rohde, C. C. Glüer, and R. Barkmann, "The preliminary evaluation of a $1 \mathrm{mhz}$ ultrasound probe for measuring the elastic anisotropy of human cortical bone," Ultrasonics, vol. 54, no. 1, pp. 4-10, 2014.

[19] M. Gräsel, C. C. Glüer, and R. Barkmann, "Characterization of a new ultrasound device designed for measuring cortical porosity at the human tibia: A phantom study," Ultrasonics, vol. 76, pp. 183-191, 2017.

[20] G. Renaud, P. Kruizinga, D. Cassereau, and P. Laugier, "In vivo ultrasound imaging of the bone cortex," Physics in Medicine \& Biology, vol. 63 , no. 12 , p. $125010,2018$.

[21] J. Rho, L. Kuhn-Spearing, and P. Zioupos, "Mechanical properties and the hierarchical structure of bone," Med. Eng. Phys., vol. 20, no. 2, pp. 92-102, 1998.

[22] W. J. Parnell and Q. Grimal, "The influence of mesoscale porosity on cortical bone anisotropy. investigations via asymptotic homogenization," Journal of The Royal Society Interface, vol. 6, no. 30, pp. 97-109, 2009.

[23] D. Rohrbach, S. Lakshmanan, F. Peyrin, M. Langer, A. Gerisch, Q. Grimal, P. Laugier, and K. Raum, "Spatial distribution of tissue level properties in a human femoral cortical bone," Journal of Biomechanics, vol. 45, no. 13, pp. 2264-2270, 2012.

[24] X. Cai, L. Peralta, R. Brenner, G. Iori, D. Cassereau, K. Raum, P. Laugier, and Q. Grimal, "Anisotropic elastic properties of human cortical bone tissue inferred from inverse homogenization and resonant ultrasound spectroscopy," Materialia, vol. 11, p. 100730, 2020.

[25] L. Thomsen, "Weak elastic anisotropy," Geophysics, vol. 51, no. 10, pp. 1954-1966, 1986.

[26] B. Treeby, T. Varslot, E. Zhang, J. Laufer, and P. Beard, "Automatic sound speed selection in photoacoustic image reconstruction using an autofocus approach.” J Biomed Opt, vol. 16, no. 9, p. 090501, 2011.

[27] L. Nock, G. Trahey, and S. Smith, "Phase aberration correction in medical ultrasound using speckle brightness as a quality factor," $J$. Acoust. Soc. Am., vol. 85, no. 5, pp. 1819-1833, 1989.

[28] G. Trahey, D. Zhao, J. Miglin, and S. Smith, "Experimental results with a real-time adaptive ultrasonic imaging system for viewing through distorting media," IEEE Transactions on Ultrasonics, Ferroelectrics, and Frequency Control, vol. 37, no. 5, pp. 418-427, 1990.

[29] M. E. Anderson, M. S. McKeag, and G. E. Trahey, "The impact of sound speed errors on medical ultrasound imaging," J. Acoust. Soc. Am., vol. 107, no. 6, pp. 3540-3548, 2000.

[30] H. S. Yoon and J. L. Katz, "Ultrasonic wave propagation in human cortical bone-ii. measurements of elastic properties and microhardness." J Biomech, vol. 9, no. 7, pp. 459-464, 1976.

[31] M. Granke, Q. Grimal, A. Saïed, P. Nauleau, F. Peyrin, and P. Laugier, "Change in porosity is the major determinant of the variation of cortical bone elasticity at the millimeter scale in aged women," Bone, vol. 49, no. 5, pp. 1020-1026, 2011.

[32] S. Bernard, J. Schneider, P. Varga, P. Laugier, K. Raum, and Q. Grimal, "Elasticity-density and viscoelasticity-density relationships at the tibia mid-diaphysis assessed from resonant ultrasound spectroscopy measurements." Biomech Model Mechanobiol, vol. 15, no. 1, pp. 97-109, 2016.

[33] B. Auld, Acoustic fields and waves in solids - Volume 1. John Wiley and Sons, 1973.

[34] X. Cai, L. Peralta, A. Giron, L. Helfen, C. Olivier, F. Peyrin, P. Laugier, and Q. Grimal, "Cortical bone elasticity measured by resonant ultrasound spectroscopy is not altered by defatting and synchrotron x-ray imaging," Journal of the Mechanical Behavior of Biomedical Materials, vol. 72, pp. 241-245, 2017.

[35] X. Cai, H. Follet, L. Peralta, M. Gardegaront, D. Farlay, R. Gauthier, B. Yu, E. Gineyts, C. Olivier, M. Langer, A. Gourrier, D. Mitton, F. Peyrin, Q. Grimal, and P. Laugier, "Anisotropic elastic properties of human femoral cortical bone and relationships with composition and microstructure in elderly," Acta Biomaterialia, vol. 90, pp. 254-266, 2019.

[36] G. Renaud, J. L. Johnson, and D. Cassereau, "Real-time kirchhoff migration for ultrasound imaging of the bone cortex," SEG Technical Program Expanded Abstracts, pp. 4797-4801, 2018.

[37] J. Jensen, S. Nikolov, K. Gammelmark, and M. Pedersen, "Synthetic aperture ultrasound imaging," Ultrasonics, vol. 44, Supplement, pp. e5 - e15, 2006.

[38] B. Yiu, I. Tsang, and A. Yu, "Gpu-based beamformer: Fast realization of plane wave compounding and synthetic aperture imaging," IEEE Trans. UFFC, vol. 58, no. 8, pp. 1698-1705, 2011. 
[39] W. Press, S. Teukolsky, W. Vetterling, and B. Flannery, Numerical Recipes in C++: The Art of Scientific Computing. Cambridge University Press, 2002.

[40] H. Chauris and M. Noble, "Two-dimensional velocity macro model estimation from seismic reflection data by local differential semblance optimization: applications to synthetic and real data sets," Geophysical Journal International, vol. 144, no. 1, pp. 14-26, 2001.

[41] B. Hartmann and J. Jarzynski, "Immersion apparatus for ultrasonic measurements in polymers," J. Acoust. Soc. Am., vol. 56, no. 5, pp. 1469-1477, 1974.

[42] S. Bernard, Q. Grimal, and P. Laugier, "Resonant ultrasound spectroscopy for viscoelastic characterization of anisotropic attenuative solid materials," The Journal of the Acoustical Society of America, vol. 135, no. 5, pp. 2601-2613, 2014.

[43] J. Foiret, J. G. Minonzio, C. Chappard, M. Talmant, and P. Laugier, "Combined estimation of thickness and velocities using ultrasound guided waves: a pioneering study on in vitro cortical bone samples," IEEE Trans. UFFC, vol. 61, no. 9, pp. 1478-1488, 2014.

[44] C. M. Moran, N. L. Bush, and J. C. Bamber, "Ultrasonic propagation properties of excised human skin." Ultrasound Med Biol, vol. 21, no. 9, pp. 1177-1190, 1995.

[45] E. Perilli, Y. Bala, R. Zebaze, K. J. Reynolds, and E. Seeman, "Regional heterogeneity in the configuration of the intracortical canals of the femoral shaft," Calcified Tissue International, vol. 97, no. 4, pp. 327-335, 2015.

[46] A. A. Espinoza Orias, J. M. Deuerling, M. D. Landrigan, J. E. Renaud, and R. K. Roeder, "Anatomic variation in the elastic anisotropy of cortical bone tissue in the human femur," Journal of the Mechanical Behavior of Biomedical Materials, vol. 2, no. 3, pp. 255-263, 2009.

[47] J. Heřt, P. Fiala, and M. Petrtýl, "Osteon orientation of the diaphysis of the long bones in man," Bone, vol. 15, no. 3, pp. 269-277, 1994.

[48] M. Petrtýl, J. Heřt, and P. Fiala, "Spatial organization of the haversian bone in man," Journal of Biomechanics, vol. 29, no. 2, pp. 161-169, 1996.

[49] C. Hennig, C. D. L. Thomas, J. G. Clement, and D. M. L. Cooper, "Does 3d orientation account for variation in osteon morphology assessed by 2d histology?" Journal of Anatomy, vol. 227, no. 4, pp. 497-505, 2015.

[50] J.-G. Minonzio, N. Bochud, Q. Vallet, D. Ramiandrisoa, A. Etcheto, K. Briot, S. Kolta, C. Roux, and P. Laugier, "Ultrasound-based estimates of cortical bone thickness and porosity are associated with nontraumatic fractures in postmenopausal women: A pilot study," Journal of Bone and Mineral Research, vol. 34, no. 9, pp. 1585-1596, 2019.

[51] J. Etgen, S. Gray, and Y. Zhang, "An overview of depth imaging in exploration geophysics," Geophysics, vol. 74, no. 6, pp. WCA5-WCA17, 2009.

[52] G. Kino, Acoustic waves: devices, imaging and analog signal processing. Prentice Hall, 1987.

[53] D. Verschuur, Seismic multipal removal techniques: past, present and future (Revised Edition). EAGE Publications, 2013.

[54] P. M. Shearer, Introduction to Seismology. Cambridge: Cambridge University Press, 2009.

[55] C. Holmes, B. Drinkwater, and P. Wilcox, "Post-processing of the full matrix of ultrasonic transmit-receive array data for non-destructive evaluation," NDT \& E International, vol. 38, no. 8, pp. 701-711, 2005.

[56] P. Podvin and I. Lecomte, "Finite difference computation of traveltimes in very contrasted velocity models: a massively parallel approach and its associated tools," Geophysical Journal International, vol. 105, no. 1, pp. 271-284, 1991.

[57] L. Brekhovskikh and O. Godin, Acoustics of Layered Media II: Point Sources and Bounded Beams. Wave Phenomena. Springer Verlag, Berlin (Vol. 10), 1992.

[58] E. Bossy, M. Talmant, and P. Laugier, "Effect of bone cortical thickness on velocity measurements using ultrasonic axial transmission: A 2d simulation study," J. Acoust. Soc. Am., vol. 112, no. 1, pp. 297-307, 2002.

[59] E. Bossy, M. Talmant, M. Defontaine, F. Patat, and P. Laugier, "Bidirectional axial transmission can improve accuracy and precision of ultrasonic velocity measurement in cortical bone: a validation on test materials," IEEE Transactions on Ultrasonics, Ferroelectrics, and Frequency Control, vol. 51, no. 1, pp. 71-79, 2004.

[60] W. Telford, L. Geldart, and R. Sheriff, Applied Geophysics (Second edition) Chap. 4. Cambridge University Press, 1990.

[61] N. S. Neidell and M. T. Taner, "Semblance and other coherency measures for multichannel data," Geophysics, vol. 36, no. 3, pp. 482-497, 1971.

[62] E. Dijkstra, "A note on two problems in connexion with graphs," Numerische Mathematik, vol. 1, no. 1, pp. 269-271, 1959.

[63] D. Hong, Medical Image Segmentation Based on Accelerated Dijkstra Algorithm. Berlin, Heidelberg: Springer Berlin Heidelberg, 2012, pp. 341-348. 